\title{
Neutron Crystallography Data Collection and Processing for Modelling Hydrogen Atoms in Protein Structures
}

\author{
Gabriela C. Schröder $^{1,2}$, Flora Meilleur ${ }^{1,2}$ \\ ${ }^{1}$ Department of Molecular and Structural Biochemistry, North Carolina State University ${ }^{2}$ Neutron Scattering Division, Oak Ridge National Laboratory
}

\section{Corresponding Author}

Flora Meilleur

fmeille@ncsu.edu

\section{Citation}

Schröder, G.C., Meilleur, F. Neutron Crystallography Data Collection and Processing for Modelling Hydrogen Atoms in Protein Structures. J. Vis. Exp. (166), e61903, doi:10.3791/61903 (2020).

\section{Date Published}

December 1, 2020

\section{DOI}

$10.3791 / 61903$

URL

jove.com/video/61903

\section{Abstract}

Neutron crystallography is a structural technique that allows determination of hydrogen atom positions within biological macromolecules, yielding mechanistically important information about protonation and hydration states while not inducing radiation damage. X-ray diffraction, in contrast, provides only limited information on the position of light atoms and the X-ray beam rapidly induces radiation damage of photosensitive cofactors and metal centers. Presented here is the workflow employed for the IMAGINE and MaNDi beamlines at Oak Ridge National Laboratory (ORNL) to obtain a neutron diffraction structure once a protein crystal of suitable size $\left(>0.1 \mathrm{~mm}^{3}\right)$ has been grown. We demonstrate mounting of hydrogenated protein crystals in quartz capillaries for neutron diffraction data collection. Also presented is the vapor exchange process of the mounted crystals with $\mathrm{D}_{2} \mathrm{O}$-containing buffer to ensure replacement of hydrogen atoms at exchangeable sites with deuterium. The incorporation of deuterium reduces the background arising from the incoherent scattering of hydrogen atoms and prevents density cancellation caused by their negative coherent scattering length. Sample alignment and room temperature data collection strategies are illustrated using quasi-Laue data collection at IMAGINE at the High Flux Isotope Reactor (HFIR). Furthermore, crystal mounting and rapid freezing in liquid nitrogen for cryo-data collection to trap labile reaction intermediates is demonstrated at the MaNDi time-offlight instrument at the Spallation Neutron Source (SNS). Preparation of the model coordinate and diffraction data files and visualization of the neutron scattering length density (SLD) maps will also be addressed. Structure refinement against neutron dataonly or against joint X-ray/neutron data to obtain an all-atom structure of the protein of interest will finally be discussed. The process of determining a neutron structure will be demonstrated using crystals of the lytic polysaccharide monooxygenase Neurospora crassa LPMO9D, a copper-containing metalloprotein involved in the degradation of recalcitrant polysaccharides via oxidative cleavage of the glycosidic bond. 


\section{Introduction}

Neutron macromolecular crystallography is a technique that provides a unique window into the structure and underlying chemistry of proteins. Conceptually similar to Xray diffraction, neutron diffraction provides atomistic details of macromolecular structure, however, the interaction of neutrons with nuclei enables localization of light atoms, often difficult to detect with X-ray diffraction ${ }^{1}$. During X-ray diffraction, X-rays scatter from the electron cloud, making light atoms such as hydrogen $(\mathrm{H})$ poorly visible in electron density maps that do not have near sub-Ångström resolution ${ }^{2}$. In contrast, the scattering intensity of neutrons depends on complex interactions with the nucleus, with isotopes of the same element displaying different scattering lengths. Therefore, light atoms and their isotopes, such as hydrogen $\left({ }^{1} \mathrm{H}\right)$ and deuterium $\left({ }^{2} \mathrm{H}\right.$ or $\left.\mathrm{D}\right)$, have comparable visibility to the backbone carbon, nitrogen and oxygen atoms in neutron scattering length density (SLD) maps. Furthermore, since the magnitude of neutron scattering is independent of the number of electrons, scattering from light elements is not obscured by heavy elements when they are in close vicinity to each other, as is observed in X-ray scattering. The enhanced visibility of $\mathrm{H}$ and its isotope $\mathrm{D}$ when employing neutron diffraction provides valuable information about the protonation state of catalytically important residues, cofactors and ligands and aids the orientation of water molecules, revealing important information about catalytic mechanisms and protein chemistry ${ }^{3}$. Neutron diffraction also offers the advantage of being a non-destructive technique, particularly suited to biological samples sensitive to ionization such as proteins with metal centers or photosensitive redox cofactors $^{2}$. The primary focus of this article is to provide an overview of the workflow to obtain a high-quality neutron protein crystal structure. We refer the interested reader to Podjarny et al. ${ }^{4}$, Blakeley ${ }^{5}$, Blakeley et al. ${ }^{6}$ and O'Dell et al. ${ }^{3}$ for an excellent overview of neutron protein diffraction and Ashkar et al. ${ }^{7}$ for further applications of neutron scattering.

Neutrons are primarily generated during nuclear reactions employing either of two processes: nuclear fission at reactor sources or spallation at accelerator-based sources ${ }^{8}$. Reactor sources provide a continuous neutron beam by employing nuclear fission of the ${ }^{235} \mathrm{U}$ isotope while spallation neutron sources produce a pulsed neutron beam by bombarding a target, for example a liquid metal such as mercury, with protons ${ }^{9}$. Oak Ridge National Laboratory (ORNL) in Oak Ridge, Tennessee, hosts both a steady-state neutron source at the High Flux Isotope Reactor (HFIR) and a $60 \mathrm{~Hz}$ pulsed source at the Spallation Neutron Source (SNS). The IMAGINE beamline, located at the HFIR, is a neutron diffractometer optimized for biological macromolecules (Supplementary Figure 1$)^{10}$. IMAGINE employs a neutron image plate detector to measure quasiLaue data using a narrow bandpass in the range of 2.8 - $4.5 \AA$ from single crystals with unit cell edges $<150$ A. The Macromolecular Neutron Diffractometer (MaNDi), located at the SNS, is a time-of-flight (TOF) Laue neutron diffractometer equipped with a spherical detector array frame (DAF) (Supplementary Figure 2) ${ }^{11}$. MaNDi measures data from single crystals with unit cell edges in the range of 10 - $300 \AA$ by employing a tunable $2 \AA$-wavelength bandwidth between $2.0-6.0 \AA^{12}$.

The process of generating neutrons is highly energy intensive, resulting in relatively weak neutron beam fluxes when contrasted to X-ray beam fluxes at synchrotron 
sources $^{13}$. To ensure sufficient signal-to-noise ratios during data collection, it is necessary to grow crystals of suitable size and quality ${ }^{14}$. Typically, crystals with volumes $>0.1$ $\mathrm{mm}^{3}$ are needed to collect data with adequate statistics ${ }^{15}$. In addition to lower fluxes, inherent properties of the interaction between neutrons and the sample nuclei must be taken into consideration ${ }^{16}$. The scattering length of neutrons differs for isotopes of the same element, a property which can be advantageously exploited in small angle neutron scattering (SANS) to mask or highlight regions of a sample - a process known as contrast matching ${ }^{17}$. In diffraction experiments, the negative coherent neutron scattering length of $\mathrm{H}\left(-3.741 \mathrm{fm}\right.$ for $\left.{ }^{1} \mathrm{H}\right)$ can lead to cancellation of neutron scattering density map features since the coherent neutron scattering lengths of other biologically relevant atoms, including carbon $\left(6.6511 \mathrm{fm}\right.$ for $\left.{ }^{12} \mathrm{C}\right)$, nitrogen $\left(9.37 \mathrm{fm}\right.$ for $\left.{ }^{14} \mathrm{~N}\right)$, oxygen (5.803 fm for $\left.{ }^{16} \mathrm{O}\right)$, phosphorus $\left(5.13 \mathrm{fm}\right.$ for $\left.{ }^{31} \mathrm{P}\right)$ and sulfur $\left(2.804 \mathrm{fm}\right.$ for $\left.{ }^{32} \mathrm{~S}\right)$, are positive (Table 1$)^{12,14}$. Furthermore, the large incoherent scattering length of $\mathrm{H}(25.274 \mathrm{fm})$, increases the background during data collection, hampering the quality of the dataset and compromising data resolution ${ }^{7}$. To circumvent these limitations introduced by $\mathrm{H}$ it is necessary, for neutron diffraction, to exchange $H$ for its isotope deuterium, ${ }^{2} H(D)$, which has a positive coherent neutron scattering length (6.671 fm) and significantly lower incoherent scattering length $(4.04 \mathrm{fm})^{19}$. This can be achieved by perdeuteration, a process in which the protein is expressed by organisms grown in fully deuterated media ensuring complete incorporation of $\mathrm{D}$ at $\mathrm{H}$ sites $^{20}$. It is also possible to partially deuterate protein by replacing $\mathrm{H}$ with $\mathrm{D}$ solely at the exchangeable sites (titratable groups) while the non-exchangeable carbonbound sites remain hydrogenated ${ }^{21}$. This can be achieved by growth of hydrogenated protein crystals in deuterated mother liquor ${ }^{22}$. Most commonly however, H/D exchange of hydrogenated proteins is performed by vapor exchange following growth of suitably large crystals in $\mathrm{H}_{2} \mathrm{O}$-based buffer $^{23}$. In such cases, crystals are mounted in a quartz capillary and vapor-equilibrated with a $\mathrm{D}_{2} \mathrm{O}$-based mother liquor.

The limited neutron fluxes at neutron sources result in longer data collection times, ranging from days to several weeks ${ }^{24}$. At ORNL, both IMAGINE and MaNDi employ a narrow wavelength bandpass in the $2-6 \AA$ range to optimize data collection $^{25}$. Data can be collected at room temperature or at cryo-temperature. Cryo-data collection can potentially improve data quality and opens up the possibility for freeze-trapping catalytic intermediates. Following neutron diffraction data collection, an X-ray dataset is typically collected on the same crystal at the same temperature or on a crystal grown under identical conditions ${ }^{26}$. Data collection at the same temperature allows structure refinement to be performed against both X-ray and neutron data, preventing any potential temperature-induced artefacts such as changes in the visibility and position of waters or the occupancies of residues with alternate conformations ${ }^{27}$. Joint $X$-ray neutron data refinement increases the data-to-parameter ratio and provides the advantage of allowing the protein backbone coordinates to be refined against the X-ray data, while the neutron diffraction data is used to refine the position of the $H / D$ atoms ${ }^{28}$. This is particularly useful when using partially deuterated samples, where density cancellation due to $\mathrm{H}$ atoms at non-exchangeable sites on the protein is present. Although the number of X-ray structures far exceeds the number of neutron structures deposited in the Protein Data Bank (PDB), software packages initially designed for the refinement of X-ray data have been expanded to encompass neutron data as well $3,29,30$. Following data collection, models can be refined using refinement packages such 
as phenix.refine, CNSsolve (nCNS) or SHELXL $28,31,32,33$. During the refinement process, neutron scattering density maps can be visualized for manual fitting using СоOT$^{34}$. Following structure solution, the coordinates and the neutron and/or X-ray diffraction data files can be submitted to the PDB, who will validate and deposit the model, making it available for public access ${ }^{18,29,30}$.

Structural analysis of proteins is a multifaceted approach in which numerous techniques are used to probe their function and mechanism ${ }^{35}$. Neutron protein crystallography provides valuable chemical insights to expand on and complement findings from additional studies such as X-ray diffraction, spectroscopy, nuclear magnetic resonance (NMR) or micro crystal electron diffraction (microED) ${ }^{36}$. Neutron protein diffraction is uniquely positioned to provide insights into enzymatic mechanisms, since $\mathrm{H}$ atoms are central to their chemistry. The absence of radiation damage induced by neutrons make them a probe exceptionally suited to the study of metalloproteins ${ }^{37}$. We present here a representative example of the process of neutron protein diffraction from sample preparation to data collection, refinement and analysis (Figure 1). Crystals of sufficient size for neutron diffraction experiments have been grown of the metalloprotein Neurospora crassa LPMO9D (NcLPMO9D). NcLPMO9D is a copper-containing metalloprotein involved in the degradation of recalcitrant cellulose by oxygen atom insertion at the glycosidic bond ${ }^{38,39}$. The NcLPMO9D active site contains a mononuclear copper center within a characteristic "histidinebrace" composed of the N-terminal histidine and a second conserved histidine (Supplementary Figure 3$)^{40}$. The $\mathrm{N}$ terminal of fungal LPMOs is methylated but the posttransitional modification does not occur during recombinant expression in yeast. In the NcLPMO9D resting state, the copper center is present in a $\mathrm{Cu}^{2+}$ oxidation state and is activated by single electron reduction to $\mathrm{Cu}^{1+}$, allowing molecular oxygen to bind and be activated by rapidly being reduced to a superoxide species ${ }^{41,42}$. The overall NcLPMO9D reaction requires further addition of one electron and two protons to form the hydroxylated polysaccharide product $^{43}$. The identity of the activated oxygen species responsible for hydrogen atom abstraction (HAA) from the polysaccharide substrate has not been identified and intensive structural and computational studies are currently ongoing $^{44,45}$. Given the redox chemistry at the NcLPMO9D active site, mitigation of radiation damage is particularly pertinent. We illustrate here room temperature and cryotemperature data collection on NcLPMO9D crystals to determine the NcLPMO9D structure in the resting state and in the activated reduced form, respectively ${ }^{46}$. Emphasis will be given to protein crystal mounting, beamline instrument setup for data collection, the preparation of the data and coordinate files and the refinement steps necessary to model an all-atom neutron structure.

\section{Protocol}

\section{Crystal size evaluation}

1. Measure the size of the crystals using a microscope equipped with normal and polarized light. Select crystals with a minimum volume of $\sim 0.1 \mathrm{~mm}^{3}$ (Supplementary Figure 4).

2. Label wells with sufficiently large crystals and note the crystallization conditions used to generate these crystals.

\section{Preparation of deuterated crystallization buffer}

1. Dissolve crystallization buffer components in $\mathrm{D}_{2} \mathrm{O}$ to generate deuterated crystallization buffer. 
2. Adjust the $\mathrm{pH}$ of the buffer by calculating the $\mathrm{pD}$ of the solution using the following equation:

$\mathrm{pD}=\mathrm{pH}_{\text {meas }}+0.4(1)$

where $\mathrm{pH}_{\text {meas }}$ is the $\mathrm{pH}$ measured with a standard glass electrode. The original $\mathrm{pH}$ of the NCLPMO9D crystallization buffer was 6.0 , therefore we will use a $\mathrm{pH}_{\text {meas }}$ of 5.6 for the deuterated crystallization buffer at pD 6.0.

3. Immerse the $\mathrm{pH}$ meter electrode in $\mathrm{D}_{2} \mathrm{O}$ for ten minutes prior to use (Supplementary Figure 5).

4. Adjust the $\mathrm{pH}_{\text {meas }}$ to 5.6 by use of the base $\mathrm{NaOD}$ or the acid DCl.

\section{Crystal harvesting}

1. Place siliconized $22 \mathrm{~mm}$ round glass slides next to the crystallization tray from which crystals will be harvested. Use a clean glass slide per crystal (Figure 2A).

2. Open the sealed sandwich box containing the protein crystals in the 9-well large volume siliconized glass plate.

3. Remove 10-20 $\mu \mathrm{L}$ from the crystallization reservoir solution with a micropipette and place the solution on the glass slide (Figure 2B).

4. Harvest the crystal using a microloop of appropriate size and place the crystal in the reservoir solution drop on the glass slide to remove debris that are often harvested along with the crystal (Figure 2C and Figure 2D).

NOTE: It will be necessary to work quickly since the small-volume droplets may evaporate. Harvested crystals are also at risk of drying out when exposed to the atmosphere. It may be necessary to add some reservoir solution to the protein drop to prevent crystals from drying out in small crystallization drop volumes.

\section{Crystal mounting}

NOTE: Capillary mounting protocols vary with experimentalist preferences. To prevent damage to crystals, capillaries that need to be shortened should be scored with a cutting stone or sandpaper to ensure a smooth break.

1. Fill one end of a $2 \mathrm{~mm}$ diameter $50 \mathrm{~mm}$ length quartz capillary with reservoir buffer by capillary action or by directly pipetting $\sim 10 \mu \mathrm{L}$ of reservoir buffer into the capillary (Figure 3A).

NOTE: Users are encouraged to make use of quartz capillary tubes because, in addition to its mechanical strength, it is essential to limit neutron beam absorption and lower background contributions from the capillary. Glass capillaries introduce high background and absorb neutrons, compromising data quality.

2. Gently place the crystal in the reservoir buffer in the quartz capillary using the mounting loop (Figure 3B and Figure 3C).

3. Gently tap the tube to move the reservoir buffer and the therein immersed crystal down the capillary (Figure 3D).

4. Place the crystal no closer than $13.5 \mathrm{~mm}$ and no further $27.5 \mathrm{~mm}$ from one end of the capillary; this will be the mounting end (Supplementary Figure 6).

5. Aspirate the buffer solution around the crystal using a long thin pipette tip, leaving the crystal slightly wet. Do not touch the crystal (Supplementary Figure 7A).

6. Dry the capillary walls with a thin paper wick (Supplementary Figure 7B). 
7. Pipette $20-50 \mu \mathrm{L}$ of deuterated buffer solution into the end of the capillary opposite the mounting end (Figure 4A).

8. Melt beeswax with a heat "wand" and gently insert the capillary in this melted beeswax. Repeat until an airtight seal forms (Figure 4B and Figure 4C).

9. Pipette a very small amount of deuterated buffer, approximately $5 \mu \mathrm{L}$ in the mounting end of the capillary to act as a "heat sink" for the hot beeswax. Dip this end in melted beeswax to generate an airtight seal as described previously to form a capillary sealed on both ends (Figure 4D).

10. Inspect the mounted crystal using a microscope after mounting to ensure airtightness (Figure 4E).

11. Carefully secure the mounted crystals with Kim wipes in a container such as a 15-ml Falcon tube or Petri Dish and store horizontally at the temperature at which the crystals were grown (Figure 4F).

\section{Vapor exchange}

1. Replace the deuterated buffer with fresh buffer two days after crystal mounting.

2. Melt the wax seal furthest from the crystal with a heating loop, and use a pipette and paper wicks to remove the buffer.

3. Refill the capillary with $20-50 \mu \mathrm{L}$ of deuterated buffer solution and seal with wax.

4. Repeat the deuterated buffer replacement twice more at four day intervals to ensure that deuterated buffer vapor exchange is complete and allow vapor exchange for at least two weeks.

\section{Neutron protein diffraction}

NOTE: Readers interested in the IMAGINE beam line specifics are encouraged to consult Meilleur et al. 2013, Meilleur et al. $2018^{10,47}$.

1. Room temperature data collection at the IMAGINE beamline at HFIR

1. Sample mounting

1. Secure the capillary-mounted crystal on the goniometer with putty.

2. Mount the goniometer on the sample stick and center the crystal in the beam using the off-line alignment station.

3. Secure the sample stick on the instrument sample stage (Supplementary Figure 1B).

4. Ensure that the experimental hutch is vacated and open the beamline shutter for neutron data collection

\section{Data collection}

1. Open the data acquisition program on the beamline control computer and click on the Setup tab to set up the data collection strategy. Under Experiment Parameters type the sample name next to Sample Name and input the proposal number next to Proposal. Under Image Naming select Folder template and set the destination for the acquired data to be saved and select Image Prefix and type relevant frame name (Supplementary Figure 8). 
2. Open the Optics GUI and click on $\mathbf{2 . 7 8}$ for $\lambda_{\min }$ and $\mathbf{4 . 7 8}$ for $\lambda_{\max }$ to set the quasi-Laue range for data collection (Supplementary Figure 9).

3. Toggle to the Collect tab and under Next Scan Parameters insert the exposure time in seconds under Exposure, the number of frames under $\mathbf{N}$ Frames and the angles for data collection under $\Delta \phi / F$ rame. Name the frame to be collected under Image Prefix and initiate the data collection by clicking the Start Scan button (Supplementary Figure 10).

4. The diffracted neutrons will be detected by the Image Plate Detector. At the end of each exposure, the image plate will be read and the pattern displayed on the data acquisition GUI (Supplementary Figure 11).

5. Frames are indexed, integrated, wavelength normalized and scaled using Lauegen, Lscale $^{50}$ and Scala by the responsible beamline scientist, who will provide the user with the merged reflection file following data collection (Supplementary Figure 12).

NOTE: Data collection at both IMAGINE and MaNDi will be performed in quasi-Laue mode, using methodology and software developed for Laue diffraction data collection as developed by Helliwell et al. ${ }^{48}$ and Nieh et al. ${ }^{49}$.

6. Collect a corresponding X-ray dataset on the same crystal at the same temperature following neutron diffraction data collection (Supplementary Figure 13).

NOTE: A crystal grown from the same drop or under the same crystallization conditions can also be used for collecting X-ray diffraction data for joint neutron/X-ray refinement.

2. Cryo data collection at the MaNDi beamline at the SNS NOTE: Readers interested in the beam line specifics are encouraged to consult Coates et al. (2015), Meilleur et al. $2018^{10,11}$.

1. Sample mounting

1. Prepare the deuterated ascorbate soaking solution for the reduction of the crystals and the deuterated cryoprotectant. Place $20 \mu \mathrm{L}$ drops of each of these solutions into sitting drop wells in a crystallization plate.

NOTE: The cryoprotectant solution is usually the cryoprotectant that has proven effective for cryo-temperature X-ray diffraction data collection prepared in $\mathrm{D}_{2} \mathrm{O}$. This cryoprotectant can be further optimized (e.g. concentration) for neutron data collection if necessary.

2. Select loops for sample mounting and attach them to a magnetic cryo-base following the MaNDi guidelines (Supplementary Figure 14).

3. Fill a foam cryo-Dewar with liquid nitrogen. Place a metal cryo-protection sleeve within the liquid nitrogen to cool (Supplementary Figure 15A).

4. Remove the wax plugs from both ends of the capillary and tap the capillary to move the buffer plug so that the mounted crystal is immersed in buffer. Wash the crystal out into the $20 \mu \mathrm{L}$ reservoir solution drop in the sitting drop well (Supplementary Figure 15B and Supplementary Figure 15C). 
NOTE: This step is not required if $H / D$ exchange was performed by equilibration of the crystallization drops against deuterated buffer or by direct soaking of the crystal in deuterated buffer.

5. Immerse the crystal in the ascorbate soaking solution for two hours. Mount the crystal in a microloop attached to a magnetic cryobase. Immerse the mounted crystal in the cryoprotectant for 10 seconds and plunge the crystal and cryo mount into the liquid nitrogen to freeze (Supplementary Figure 15D).

6. Once the crystal is frozen, use pre-cooled cryo pin tong and mount the crystal on the MaNDi sample stage fitted with a cryo-stream. Gently open the cryo pin tong and ensure that the crystal remains in the cryo-stream (Supplementary Figure 2C).

\section{Data collection}

1. Open the data collection software in which the experiment information will have autopopulated.

2. Click on the center of the crystal to center it with the computer-controlled goniometer (Supplementary Figure 16).

3. Under Table, set up the data collection strategy by inputting the angles for data collection under "phi" as well as the total neutron beam exposure per frame under Value (Supplementary Figure 17).

4. Click on Submit to start data collection.
5. As data collects, diffracted neutron will be visible (Supplementary Figure 17). Diffraction spots will become clearer as the exposure time increases, improving the signal-to-noise ratio (Figure 5).

6. Frames will be indexed, integrated, wavelength normalized and scaled using Mantid and Lauenorm by the responsible beamline scientist, who will provide the user with the merged intensity file following data collection ${ }^{51}$.

\section{Structure refinement}

1. Joint X-ray and neutron data refinement

1. Structure preparation

1. Refine the X-ray data to obtain a protein structure using the phenix.refine software package and Coot for manual building to obtain a completed structure.

2. Open CCP4 and select the Convert to/modify/ extend MTZ program to match the R-free data flags of the neutron data to those of the X-ray data. Select to import the reflection file in MTZ format. Under In upload the obtained neutron MTZ file and upload the X-ray mtz file under Import FreeR MTZ (Supplementary Figure 18). Give a name for the new MTZ file under Out and click Run.

3. Open the Phenix software package and click on ReadySet under Refinement Tools. Next to PDB file upload the PDB coordinate file refined against the X-ray data. Select to Add hydrogens to model if absent and select $\mathbf{H}$ / $D$ at exchangeable sites, $\mathrm{H}$ elsewhere from 
the drop-down menu. Select Add deuteriums

to solvent molecules and leave the remaining options on their default values (Supplementary Figure 19A).

NOTE: If a perdeuterated protein is used, select the option Add hydrogens to model if absent and select H/D at exchangeable sites, D elsewhere.

2. Structure refinement

1. Open the phenix.refine program under the Refinement tab to set up the refinement using both X-ray and neutron data. In the Configure tab in Input files input the PDB file from the X-ray structure which has been processed with ReadySet and the necessary CIF restrains file for any relevant ligands. Upload the MTZ file from the neutron data with the Rfree flags assigned using CCP4 and assign this as "Neutron data" and "Neutron R-free" under the Data type heading. Upload the MTZ file from the X-ray data and assign this as " $X$ ray data" and "X-ray R-free" under the Data type heading. The Space group and Data labels will auto-populate once the data has been uploaded (Supplementary Figure 19B).

NOTE: When performing the refinement, and inputting the crystal information, use the unit cell determined from the X-ray data.

2. Under the Configure tab in the Refinement settings keep the standard refinement strategy. Increase the number of cycles to five (Supplementary Figure 20)
3. Select All parameters, click on Advanced and select Hydrogens.... Change the Hydrogen refinement model to individual and turn off the Force riding adp (Supplementary Figure 20).

4. Select All parameters and open the Search parameters option. Search for the word nuclear and select to Use the nuclear distances for X-H/D (Supplementary Figure 20).

5. Select Run to initiate the refinement.

3. Model building

1. Following the refinement in Phenix, click on Open in Coot in the Results tab to visualize the X-ray electron density and neutron SLD maps. Click on the Display Manager tab and under Maps click on Delete Map next to the _neutron maps to remove the neutron maps (Supplementary Figure 21). Click on File > Open MTZ, mmCIF fcf or phs.... Select the current refinement files and open the .mtz file. For both the Amplitudes and Phases option select the 2FoFCWT_no_fill_neutron data from the dropdown menu. Repeat this and open the FOFCWT_neutron data. Open Display Manager and toggle to Scroll for both the neutron and $\mathrm{X}$-ray 2FoFCWT maps then scroll to decrease the rmsd of the $2 \mathrm{FOF}_{\mathrm{CWT}}$ maps to 1.00 (Supplementary Figure 21). Toggle to Scroll for both the neutron and X-ray FOFCWT maps and scroll to decrease the rmsd of the FOFCWT maps to 3.00 . 
2. Perform visual inspection of the residues to determine whether the model fits the data. Determine the correct orientation and $H / D$ occupancy of all exchangeable sites by analyzing difference density map peaks. This includes the hydroxyl groups of serines, threonines and tyrosines; the nitrogen of histidine, glutamine, asparagine and lysine; the sulfhydryl of cysteine; the carboxyl groups of aspartate and glutamate; backbone amide groups; ligands; cofactors and any potential functionalized residues (Figure 6).

3. Reorient water molecules according to neutron density and hydrogen bond interactions by rotating them using the Rotate Translate Zone/ Chain/Molecule feature (Supplementary Figure 22A and Supplementary Figure 22B). Adjust positions of protein residue $H / D$ exchangeable sites using the Edit Chi Angles tool and the Rotate Translate Zone/Chain/ Molecule feature (Supplementary Figure 22C and Supplementary Figure 22D).

2. Structure refinement - neutron data-only refinement

1. Structure preparation

1. Open Phenix and select "Molecular Replacement" and select "Phaser-MR (full featured)" to derive the phase of the scaled intensities provided by the instrument scientist by molecular replacement to generate a starting coordinate file in pdb format. Input the starting pdb structure in the "Input and general options tab and complete the Ensembles, ASU contents and Search Procedure options.
2. Open Model tools and select PDB Tools. Insert the pdb file as the Input file. Navigate to the Options tab and under Remove atom selection select the names of the solvent, ligands, cofactors and metals. This removes all water molecules, cofactors, ligands and metal ions to generate a minimal model. Additionally, select to Remove alternate conformers from the model (Supplementary Figure 23).

3. Select Refinement in Phenix and open ReadySet. Input the edited pdb coordinate file next to PDB file. Select to Add hydrogens to model if absent and select $H / D$ at exchangeable sites, $\mathrm{H}$ elsewhere from the drop-down menu of Neutron refinement options (Supplementary Figure 23).

2. Structure refinement

1. Open the phenix.refine program under the Refinement tab in Phenix. In the Configure tab input the PDB file which has been processed with ReadySet under the Input files box. In the Input files box upload the MTZ file from the neutron data and assign this as X-ray data and X-ray R-free under the Data type column even though it is neutron data. The refinement configuration set up in the next step will be used to treat the reflection file as neutron data. The Space group and Data labels will autopopulate once the data has been uploaded (Supplementary Figure 24A).

2. Under the Configure tab in the Refinement settings keep the standard refinement strategy. Increase the number of cycles to five. Under 
Other options select neutron from the Scattering table dropdown menu. Deselect the option to Update waters (Supplementary Figure 24B).

3. Select

parameters $>$ Advanced $>$ Hydrogens. In the new window select Individual from the Hydrogen refinement model dropdown menu and turn off the Force riding adp (Supplementary Figure 20).

4. Select All parameters $>$ Search parameters... option. Search for the word nuclear and select to Use the nuclear distances for $X-H / D$ (Supplementary Figure 20).

5. Select to Run to initiate the refinement.

NOTE: Following the initial refinement, it will be necessary to visually inspect the neutron SLD maps and perform manual model building in Coot. It may be necessary to insert ligands/ cofactors present in the model. Subsequent refinements will require the necessary CIF restraints file for any relevant ligands and these should be uploaded in the Configure tab of phenix.refine.

\section{Model building}

1. Following the refinement in Phenix, click on Open in Coot in the Results tab to visualize the neutron SLD maps and structure. Click on the Display Manager tab and under Maps click on Delete Map to delete both the 2FoFCWT and FOFCWT maps (Supplementary Figure 25). Click on File > Open MTZ, mmCIF fcf or phs.... Select the current refinement folder and select the .mtz file. For both the Amplitudes and Phases option select the 2FoFcWT_no_fill data from the dropdown menu. Repeat by clicking on File > Open MTZ, mmCIF fcf or phs... and select the FOFCWT data from the dropdown menu for both the Amplitudes and Phases option. Open Display Manager and toggle to Scroll for the 2FoFCWT_no_fill map then scroll to decrease the rmsd of the 2FOFCWT_no_fill data to 1.00 (Supplementary Figure 25). Toggle to Scroll for the FOFCWT map and scroll to decrease the rmsd of the FOFCWT data to 3.00 .

2. Perform visual inspection of the protein structure to determine whether the model fits the neutron SLD map.

3. As described in 7.1.3.2, determine the correct orientation and H/D occupancy of residues and groups with H/D exchangeable sites. Adjust residue positions using the Rotate Translate tool and Edit Chi Angles (Figure 6). If necessary, Real Space Refine Zone can be used. Fix $\mathrm{D}$ atoms that explode from the residue manually by using the text editor to insert the correct atom coordinates

NOTE: Real Space Refine Zone is not optimized for neutron SLD maps in Coot and may result in irregular bond lengths for atoms bound to $D$, termed exploding residues (Supplementary Figure 26). It is preferable to manually edit the necessary atomic coordinates and avoid use of Real Space Refine Zone. 
4. Insert and reorient water molecules according to neutron density. To add waters in Coot select the Place atom at pointer icon and select to insert a water molecule (Supplementary Figure 27A). Coot will insert an $\mathrm{O}$ atom at this position by default.

5. To add $\mathrm{D}$ atoms to the $\mathrm{O}$ atoms of waters inserted in Coot use Phenix. Open the Refinement menu and click on ReadySet. Next to Neutron Refinement Options select only the option to Add deuteriums to solvent molecules. Deselect Add hydrogens to model if absent (Supplementary Figure 27B and Supplementary Figure 27C).

NOTE: Model building using neutron-only data differs from model building of a joint X-ray/ neutron structure because there is no $X$ ray data to contribute to the refinement of the coordinates of the backbone and heavier atoms. In a joint refinement, the electron density map is initially used to determine the protein backbone and sidechain coordinates. This model is subsequently used in a joint X-ray/ neutron data refinement in which the orientation and occupancy of $H / D$ atoms is derived from the neutron SLD map. In a neutron-only refinement, the entire structure is derived from analysis of the neutron SLD maps, requiring building of the water molecules, backbone, side-chains and ligands in addition to the H/D atoms (Figure 6). The data-to-parameter ratio is low in refinements against neutron data alone and caution should be taken not to overfit the data.

\section{Representative Results}

Neutron diffraction data on crystals of a lytic polysaccharide monooxygenase from Neurospora crassa (NcLPMO9D) were collected on IMAGINE at the HFIR at room temperature and on MaNDi at the SNS under cryo-conditions following the protocol described above. Crystals of the hydrogenated protein grown in $\mathrm{H}_{2} \mathrm{O}$-based buffer with volume greater than $0.1 \mathrm{~mm}^{3}$ were used (illustrative example of large crystals are shown in Supplementary Figure $\mathbf{4}$ and figures thereafter). Crystals were mounted in quartz capillaries and vapor exchange with the $\mathrm{D}_{2} \mathrm{O}$-based buffer was performed for three weeks prior to data collection (Figure 4).

Room temperature data collection was performed on the IMAGINE beamline (Figure 1). A four hour white-beam test led to high resolution diffraction suggesting that the crystal was of suitable size and quality for a full dataset to be collected. In addition to providing preliminary information on the diffraction quality of the crystal, the initial broad bandpass exposure can be used to index the diffraction pattern and determine the crystal orientation matrix. Given the P21 space group of the crystal, a data collection strategy of 18 frames with a collection time of 20 hours per frame was implemented. As with X-ray diffraction data collection, higher symmetry space groups require fewer frames (i.e. less angular coverage) to collect a complete dataset. The data were collected in quasi-Laue mode using a wavelength range of $2.8-4.0 \AA$. Following data collection, the data were indexed, integrated scaled and merged to give a neutron SLD file in MTZ format at a resolution of $2.14 \AA$. Data were evaluated to be of sufficient quality following similar guidelines for X-ray data analysis, although a completeness of $80 \%$ and 
a $\mathrm{CC}_{1 / 2}$ of at least 0.3 were considered acceptable since neutron protein diffraction is a flux-limited technique.

Following room-temperature neutron diffraction data collection, the same crystal was used to collect a room temperature $\mathrm{X}$-ray diffraction dataset at $1.90 \AA$ resolution (Supplementary Figure 13). The X-ray data were used to determine the positions of the "heavier" atoms including $\mathrm{C}, \mathrm{N}, \mathrm{O}$ and $\mathrm{S}$. The structure refined against the X-ray data alone was then used as the starting model to perform a joint refinement against the X-ray and neutron data. Phenix ReadySet was used to add $\mathrm{H}$ atoms at nonexchangeable sites, $H$ and $D$ atoms at exchangeable sites and $\mathrm{D}$ atoms to water molecules of the starting X-ray model. Following this model preparation, iterative refinements were performed against both datasets (Supplementary Figure 19 and Supplementary Figure 20). Interactive model building was performed in Coot by visually inspecting the density maps to orientate side-chains and water molecules accordingly (Supplementary Figure 22). The neutron data were primarily used to determine protonation states and water molecule orientations. Comparison of the electron density map of residues such as serine and tryptophan and the corresponding neutron SLD map illustrate the information that can be gained on protonation states at $H / D$ exchangeable sites from neutron protein diffraction (Figure 7). A map overlay of electron and neutron SLD maps for water molecules also indicate that while hydrogen bond interactions can be inferred from X-ray data, neutrons provide clear information regarding the orientation of these hydrogen bonds (Figure 8). Neutron SLD FO-FC omit maps were generated to determine protonation states and $H / D$ orientation of side-chains. Illustrated are the neutron SLD maps obtained for tyrosine and threonine residues, in which the neutron $\mathrm{F}_{\mathrm{O}}-\mathrm{FC}_{\mathrm{C}}$ maps clearly indicate positive peaks signifying the presence of H/D (Figure 9). The collected neutron diffraction data also provided valuable information about multiple protonation states, such as the $-\mathrm{ND}_{3}{ }^{+}$group of Lys (Figure 10). Refinement statistics ( $R_{\text {work }}$ and $\left.R_{\text {free }}\right)$ were closely monitored during model optimization to prevent over-fitting. Final statistics gave an X-ray $\mathrm{R}_{\text {work }}$ of $12.77 \%$ and an $R_{\text {free }}$ of $18.21 \%$, and a neutron $R_{\text {work }}$ of $14.48 \%$ and an $R_{\text {free }}$ of $21.41 \%$ with 389 water molecules present (Supplementary Figure 28).

Cryo-temperature data were collected on NcLPMO9D following an ascorbate soak to reduce the copper active site from $\mathrm{Cu}^{\prime l}$ to $\mathrm{Cu}^{\prime}$ on the MaNDi beamline (Supplementary Figure 2 and Supplementary Figure 15) ${ }^{45}$. Data were collected using TOF Laue mode following a neutron diffraction test using a 4 hour exposure to verify the quality of diffraction. Given the space group of the crystal, a data collection strategy of 18 frames with a collection dose of 80 Coulombs per frame was devised. The data were collected in TOF-Laue mode at a wavelength range of $2.0-4.0 \AA$. Following data collection, the data were indexed, integrated, scaled and merged to give a reflection file in MTZ format at a resolution of $2.40 \AA^{51,52}$.

Following data collection, the $2.40 \AA$ cryo-temperature NCLPMO9D neutron diffraction dataset was used for neutrononly data refinement. The neutron data were phased by molecular replacement using PDB 5TKH as the starting model. Phenix ReadySet was used to add $\mathrm{H}$ atoms at nonexchangeable sites and H/D atoms with partial occupancies at exchangeable sites. Water molecules were removed from the starting model with PDB Tools (Supplementary Figure 23). Model preparation was followed by refinement with phenix.refine using the neutron scattering table (Supplementary Figure 24). Interactive model building was performed in Coot, with water molecules being added using 
the positive peaks of the $\mathrm{FO}_{\mathrm{O}}-\mathrm{F}_{\mathrm{C}}$ map and positioned according to potential hydrogen bond interactions (Figure 11A and Figure 11B). When analyzing neutron SLD maps, water molecules are clearly visible if they are highly ordered, however their density may be spherical or ellipsoidal if they are not well-ordered (Figure 11C-E). Neutron SLD maps were used to provide valuable information on the orientation of residues such as asparagine, in which differentiating between the carbonyl and amino groups can be challenging when using X-ray diffraction data alone (Figure 12A and Figure 12B). Peaks in FO-FC neutron SLD omit maps were also very informative in determining the protonation states of histidine residues at the $\mathrm{N}_{\delta}$ - or $\mathrm{N}_{\mathcal{\varepsilon}}$-position (Figure $12 \mathrm{C}$ and Figure 12D). The protonation state of residues with multiple $H / D$ exchangeable sites can also be determined using neutron SLD maps. This was clearly illustrated with an Fo-FC neutron SLD omit map of arginine, which is known to have a positive charge (Figure 12E and Figure 12F). As previously, over-fitting was prevented by monitoring $R_{\text {work }}$ and $R_{\text {free }}$. Final statistics gave an $R_{\text {work }}$ of $22.58 \%$ and an Rfree of $30.84 \%$ (Supplementary Figure 29). Given that neutron protein diffraction is a flux limited technique in which the negative scattering length and large incoherent scattering factor of $\mathrm{H}$ must be taken in to account, it can be expected that a neutron data-only refinement would have poorer statistics than a joint X-ray/neutron-data refinement with fewer visible water molecules (Supplementary Figure 28 and Supplementary Figure 29).

When analyzing neutron SLD maps, it will become apparent that density cancellation due to the negative neutron scattering length of $\mathrm{H}$ will occur for hydrogenated proteins that were subjected to vapor exchange with $\mathrm{D}_{2} \mathrm{O}$-containing crystallization buffer. Due to this reason, neutron SLD maps in which non-exchangeable $\mathrm{H}$ atoms are attached to carbon appear incomplete when compared to their electron density map counterpart (Figure 13A). The effect of cancellation is often more apparent at poorer resolutions, making it imperative to obtain protein crystals of a high quality. It is therefore preferable to perform a joint refinement of a sample with both X-ray and neutron data in which the X-ray data can be used to determine the position of the protein backbone (Figure 13B). Furthermore, sulfur atoms in cysteine and methionine may be poorly visible, requiring X-ray data for exact atom placement (Figure 13C and Figure 13D). Metals with weak neutron scattering lengths may also be challenging to model in neutron SLD maps, as is apparent in our LPMO9D maps. Collection of a low dose (free of radiation damage) Xray dataset on the same crystal is therefore useful, since it permits metal atom positioning using electron density maps (Figure 13E and Figure 13F). 


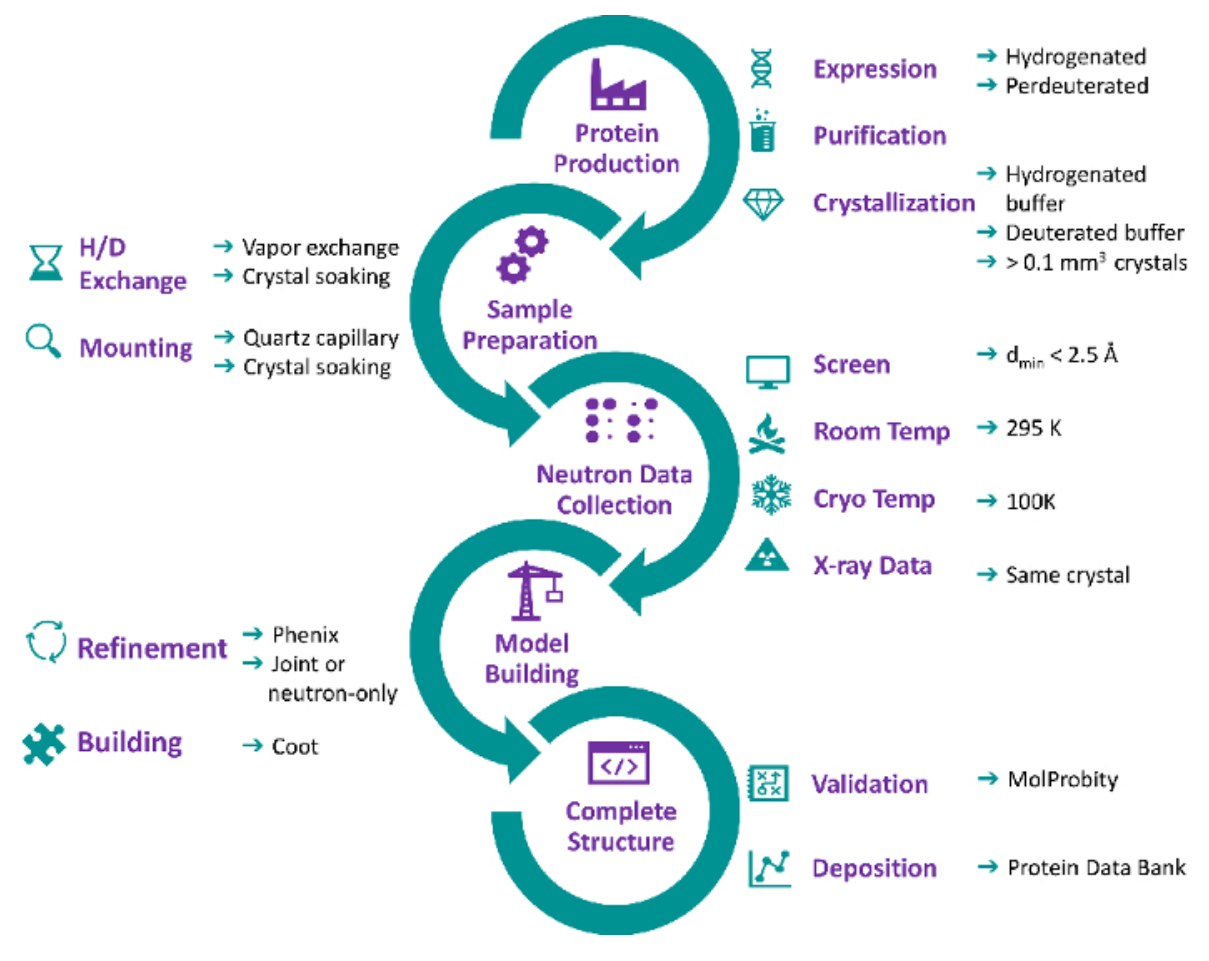

Figure 1: Flow chart of neutron protein crystallography workflow. Protein Production. In order to obtain a neutron structure, protein is first expressed. Bacterial expression in $\mathrm{H}_{2} \mathrm{O}-$ or $\mathrm{D}_{2} \mathrm{O}$-based media is typically used to produce a high yield of hydrogenated or perdeuterated recombinant protein, respectively. The protein is purified in $\mathrm{H}_{2} \mathrm{O}-$ based buffer and then crystallized in either $\mathrm{H}_{2} \mathrm{O}$ - or $\mathrm{D}_{2} \mathrm{O}$-based crystallization buffer to grow crystals to a minimum size of $0.1 \mathrm{~mm}^{3}$. Sample Preparation: Prior to neutron diffraction data collection, $\mathrm{H}_{2} \mathrm{O}$-grown crystals undergo $\mathrm{H} / \mathrm{D}$ exchange to exchange the protein titratable $\mathrm{H}$ atoms with $\mathrm{D}$. $\mathrm{H} / \mathrm{D}$ exchange can be done by direct soaking of the crystals in deuterated crystallization buffer, equilibration of the crystallization drop with a $\mathrm{D}_{2} \mathrm{O}$-based reservoir, or by mounting the crystals in quartz capillaries for vapor exchange with deuterated crystallization buffer. Neutron Data Collection: Following H/D exchange, potential crystals are screened to determine the diffraction quality. Crystals with a minimum resolution of $2.5 \AA$ are considered suitable for a full dataset to be collected. Crystals are mounted in quartz capillaries for data collection at room temperature or flash frozen in a cryo-loop for data collection at cryogenic temperature. An X-ray dataset is collected on the same (or an identical) crystal at the same temperature. Model Building: Refinement is performed using phenix.refine against both neutron and X-ray data or against the neutron data only. Manual model building of the protein structure is performed in Coot using the neutron SLD maps. Complete Structure: Following completion of the protein structure, the coordinate model is validated and deposited in the Protein Data Bank. Please click here to view a larger version of this figure. 


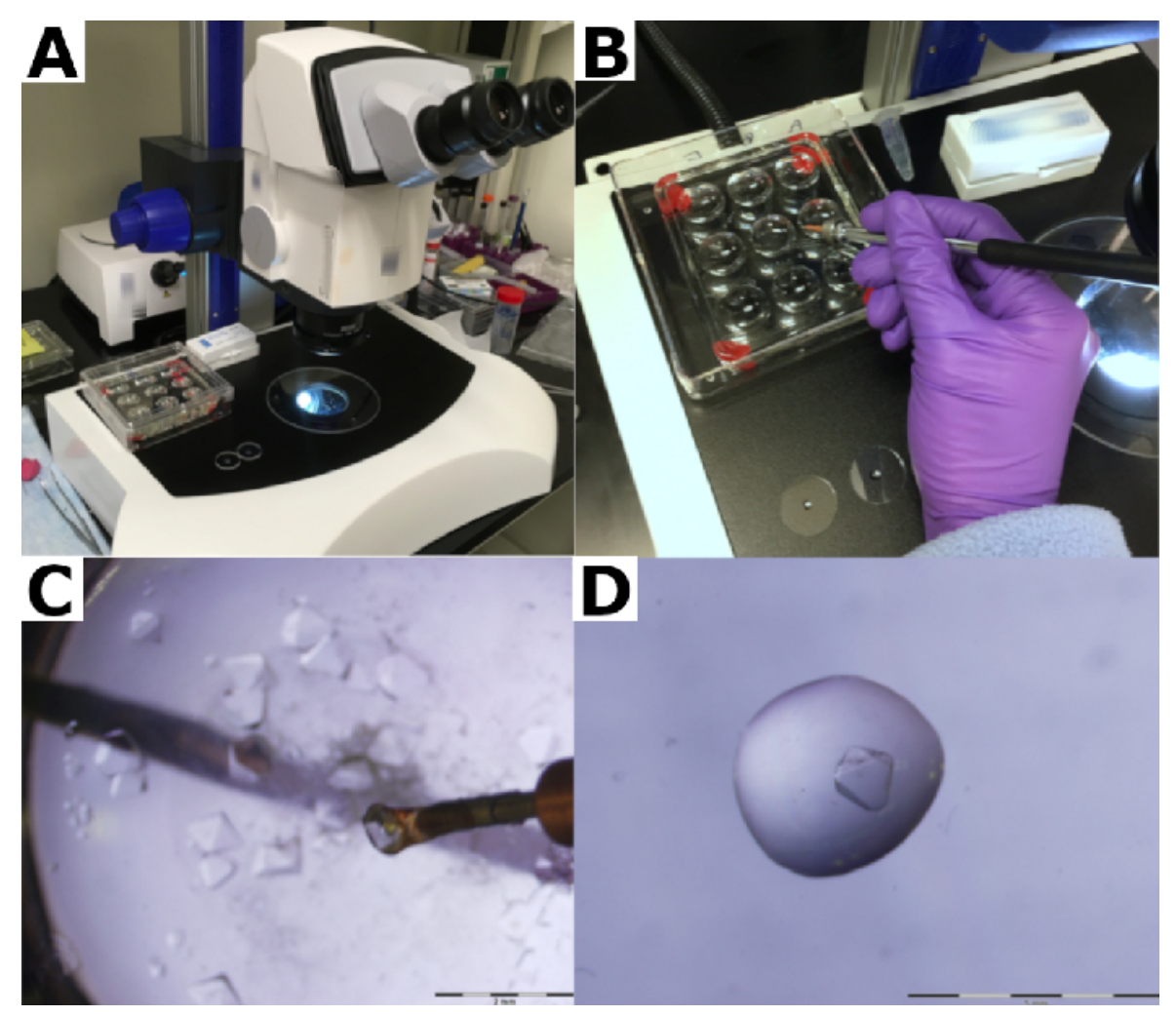

Figure 2: Harvesting protein crystals. (A) Crystals are handled under a microscope. (B) The sealed sandwich box containing the siliconized glass plate is opened. Reservoir buffer is pipetted onto siliconized glass slides. (C) A crystal is harvested with a microloop. (D) The crystal is placed in a drop of mother liquor to wash off any debris that are often harvested along with the crystal. Please click here to view a larger version of this figure. 


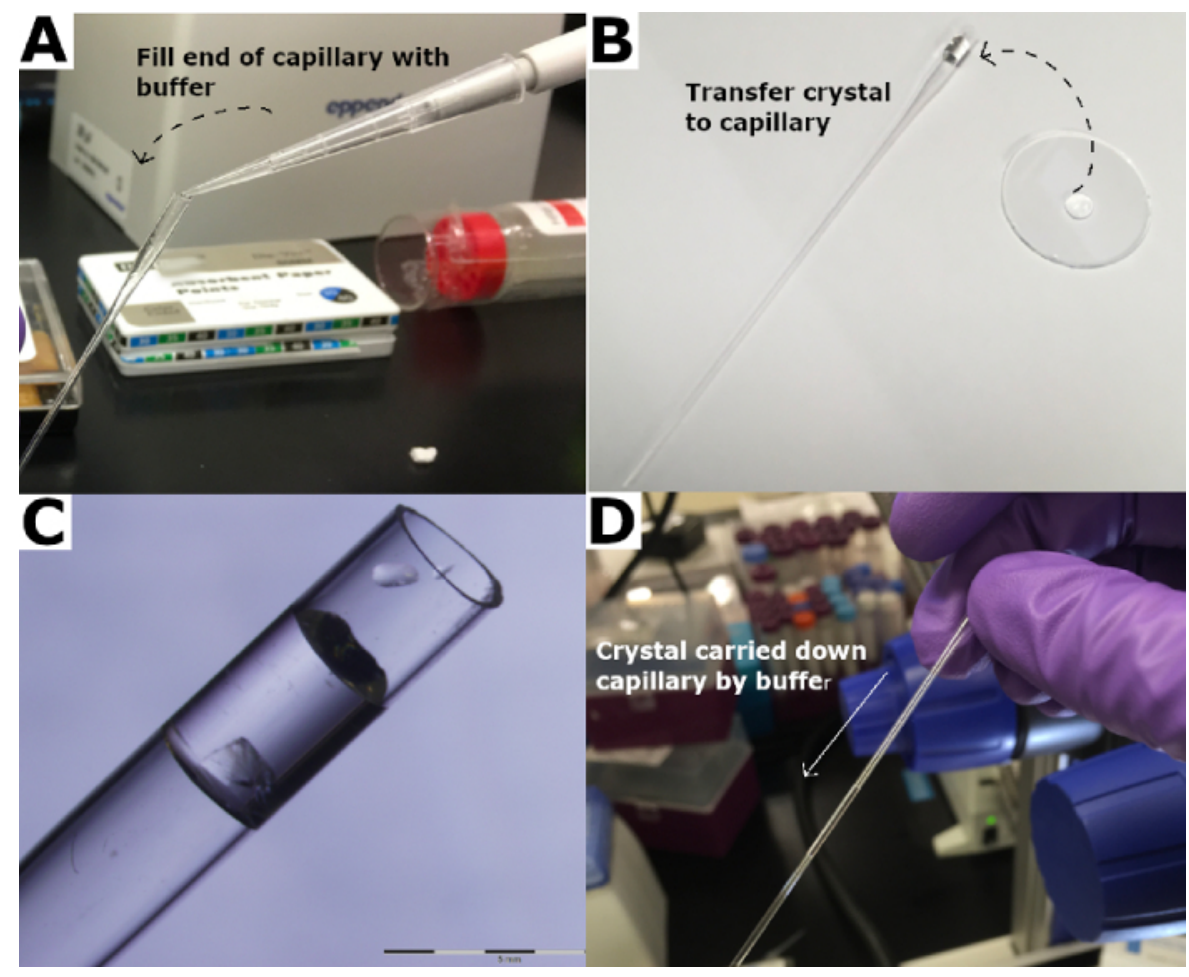

Figure 3: Transfer of crystal to quartz capillary. (A) The end of a quartz capillary is filled with reservoir buffer. (B) The crystal is transferred into the quartz capillary and (C) immersed in reservoir buffer. (D) The crystal is carried down capillary using reservoir buffer. Please click here to view a larger version of this figure. 

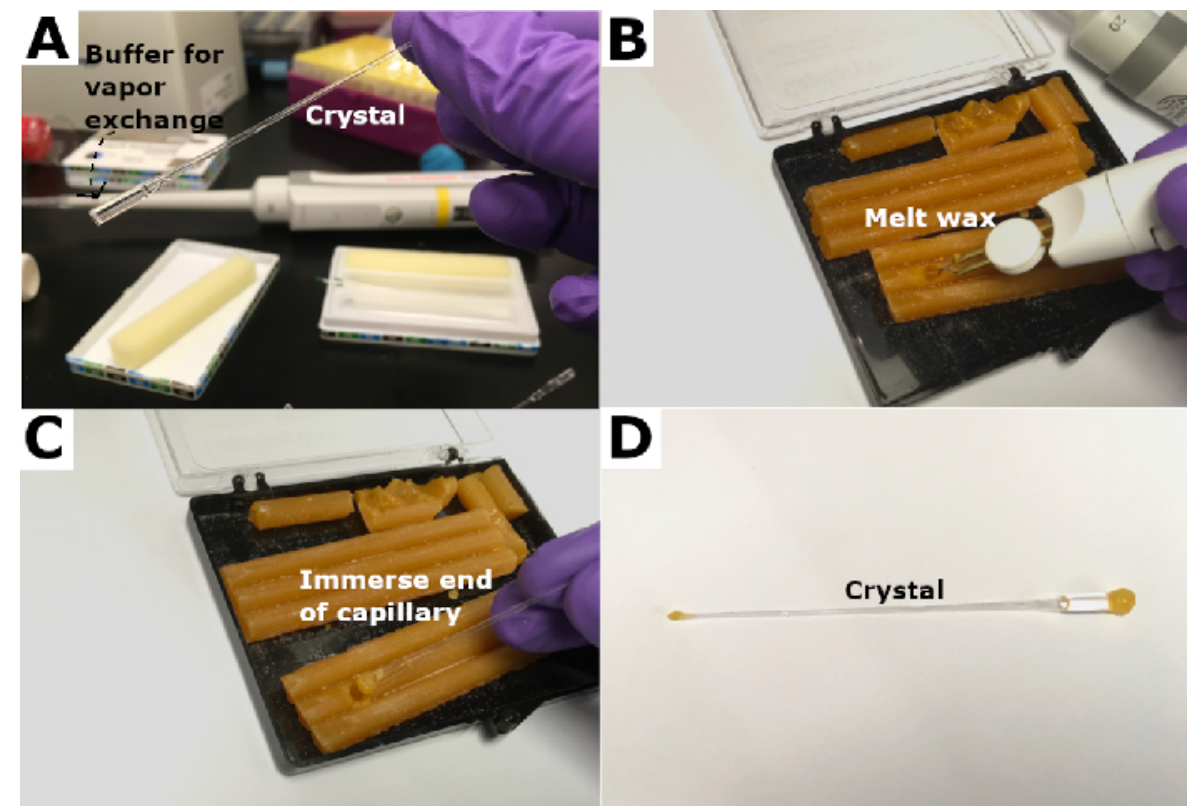

D

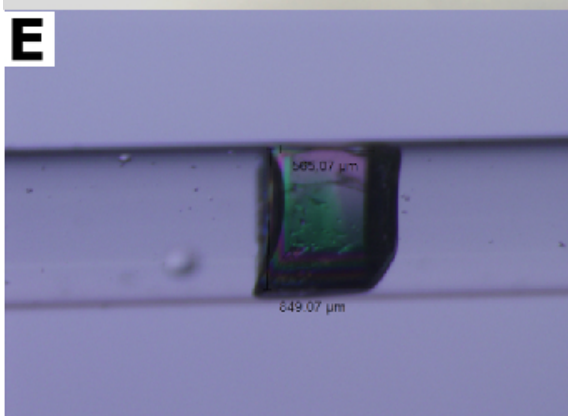

$\mathbf{F}$
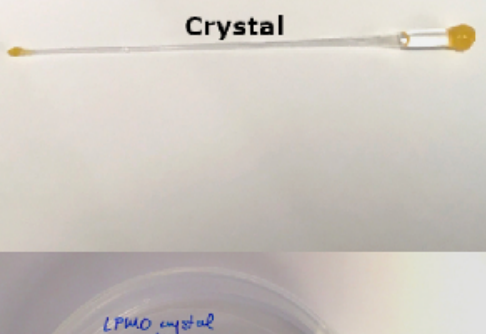
D20-butfer rapar enclenge

Figure 4: Sealing of the quartz capillary. (A) Deuterated buffer is added at the end of the capillary to form a "plug". (B) Wax is melted with a "wand". (C) The capillary is placed in the melted wax to seal. (D) Wax plugs are formed on both ends to seal the capillary. (E) The crystal after mounting. (F) The sealed capillary is placed in a Petri dish and held in place with putty. Please click here to view a larger version of this figure. 
Figure 5: Increased signal-to-noise of the neutron diffraction pattern. As data collection proceeds, diffracted spots become more intense. (NOTE: the live diffraction images presented here are for illustration and were taken from different crystals.) Please click here to view a larger version of this figure. 

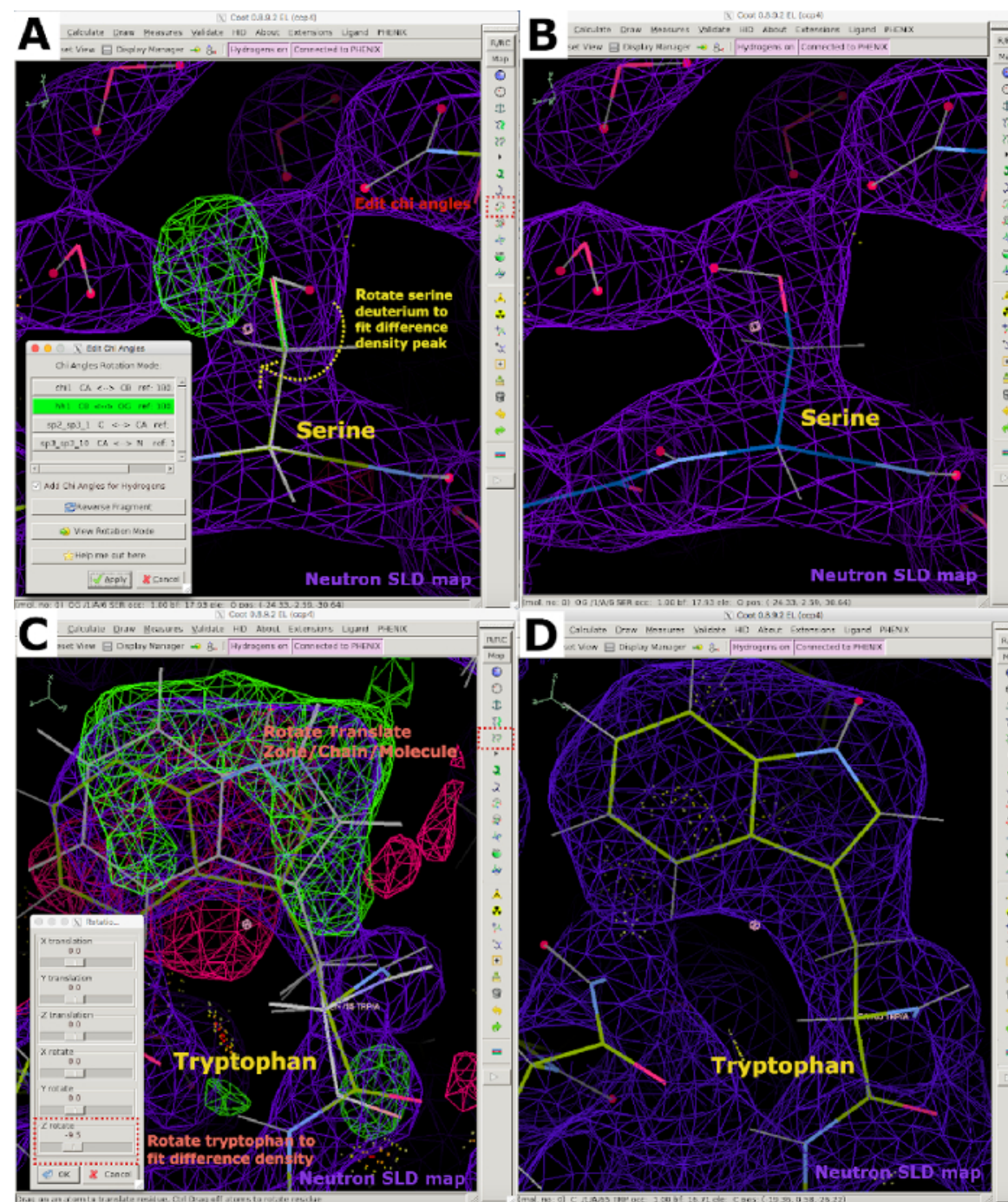

Figure 6: Interactive model building using neutron data in Coot. (A) A positive FO-FC neutron SLD density peak (green) indicating serine must be reoriented by editing chi angles. The 2FO-FC neutron SLD map is displayed in purple and 2FoFC electron density map is displayed in blue. (B) Correctly positioned serine. (C) Positive and negative FO-FC neutron SLD density peaks (green and red, respectively) indicating that tryptophan must be rotated/translated to match difference density peak. (D) Correctly oriented tryptophan. Please click here to view a larger version of this figure. 

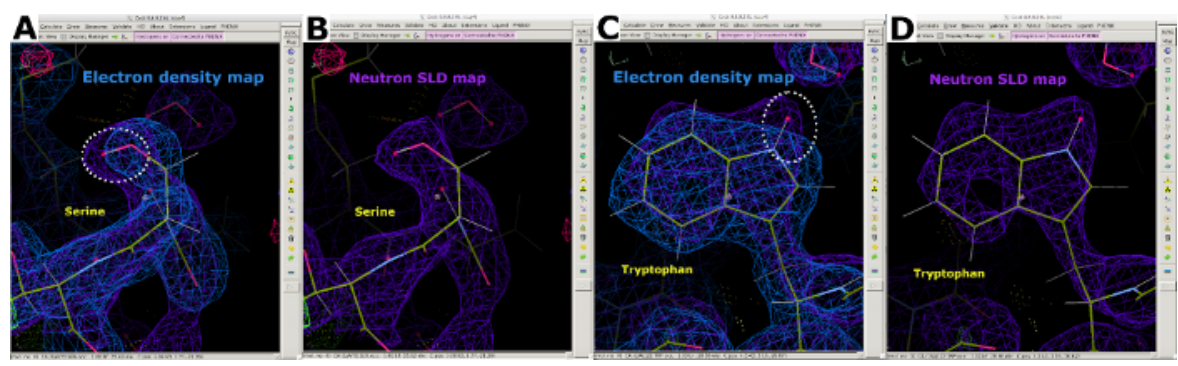

Figure 7: Additional information from neutron SLD maps. (A) 2Fo-FC electron density map (blue) displays the positions of the "heavier" atoms in serine. (B) 2FO-FC neutron SLD map (purple) clearly displays the position of the "lighter" D atom in serine. (C) 2Fo-FC electron density map (blue) displays the positions of the "heavier" atoms in tryptophan. (D) $2 \mathrm{FO}^{-} \mathrm{FC}$ neutron SLD map (purple) clearly displays the position of the "lighter" D atom in tryptophan. Please click here to view a larger version of this figure.
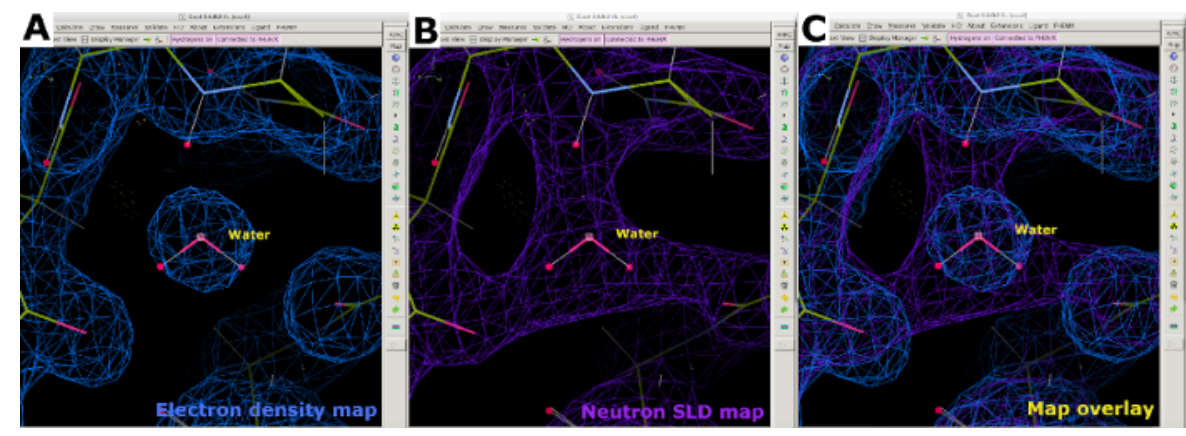

Figure 8: Water molecule positioning. (A) The spherical shape of an 2Fo-FC electron density map (blue) feature for water. (B) The $2 \mathrm{FO}_{\mathrm{O}} \mathrm{FC}_{\mathrm{C}}$ neutron SLD map (purple) provides information about the water orientation and hydrogen bond interaction. (C) Map overlay of electron and neutron SLD maps of water. The 2FO-FC neutron SLD map is displayed in purple and $2 \mathrm{FO}_{\mathrm{O}} \mathrm{FC}_{\mathrm{C}}$ electron density map is displayed in blue. Please click here to view a larger version of this figure. 

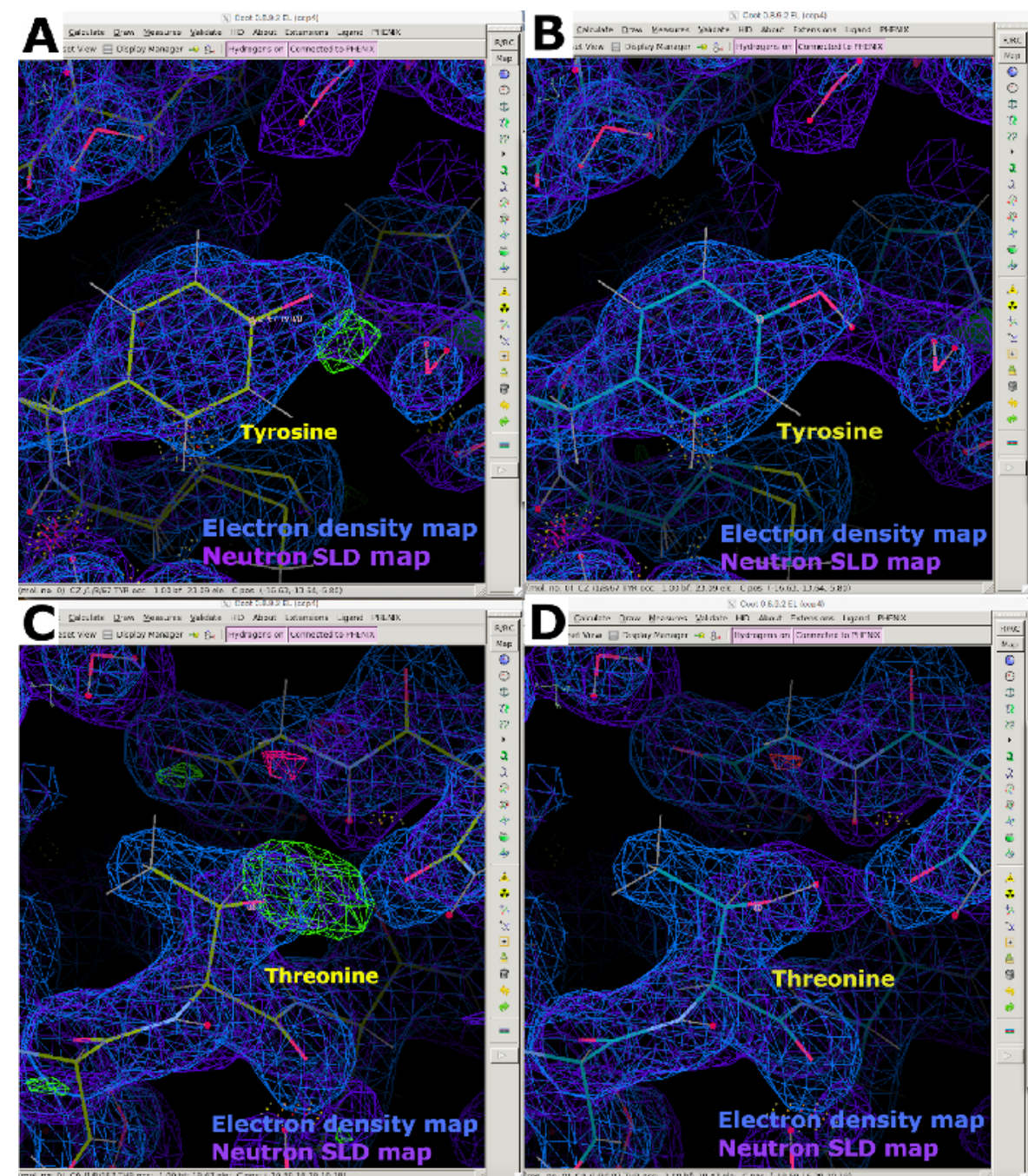

Figure 9: Neutron SLD Fo-FC omit maps. (A) The FO-FC neutron SLD map (green) provides clear information on the H/ D orientation of tyrosine residues. The $2 \mathrm{FO}_{\mathrm{O}} \mathrm{FC}_{\mathrm{C}}$ neutron SLD map is displayed in purple and $2 \mathrm{FO}_{\mathrm{O}} \mathrm{FC}_{\mathrm{C}}$ electron density map is displayed in blue. (B) Tyrosine residue with correct H/D orientation. (C) FO-FC neutron SLD map (green) provides clear information on the H/D orientation of threonine residues. (D) Threonine residue with correct H/D orientation. Please click here to view a larger version of this figure. 

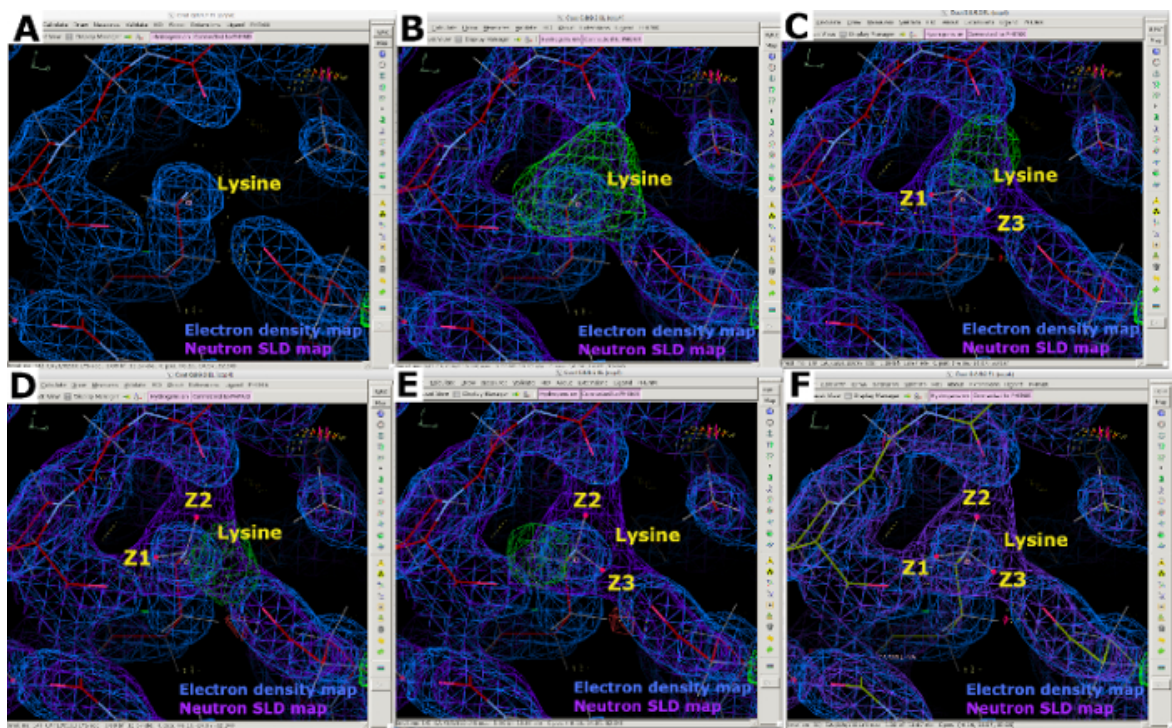

Figure 10: Multiple protonation states displayed with neutron SLD maps. (A) The $2 \mathrm{Fo}-\mathrm{FC}_{\mathrm{C}}$ electron density map (blue) only provides the position of the $\mathrm{N}$ atom of lysine $\varepsilon$-ammonium group. (B-E) The FO-FC neutron SLD omit map (green) clearly demonstrates the positively charged $-\mathrm{NH}_{3}$ group. The $2 \mathrm{FO}-\mathrm{FC}$ neutron SLD map is displayed in purple and $2 \mathrm{FO}-\mathrm{FC}$ electron density map is displayed in blue. (F) Overlay of electron density and neutron SLD maps. Please click here to view a larger version of this figure. 


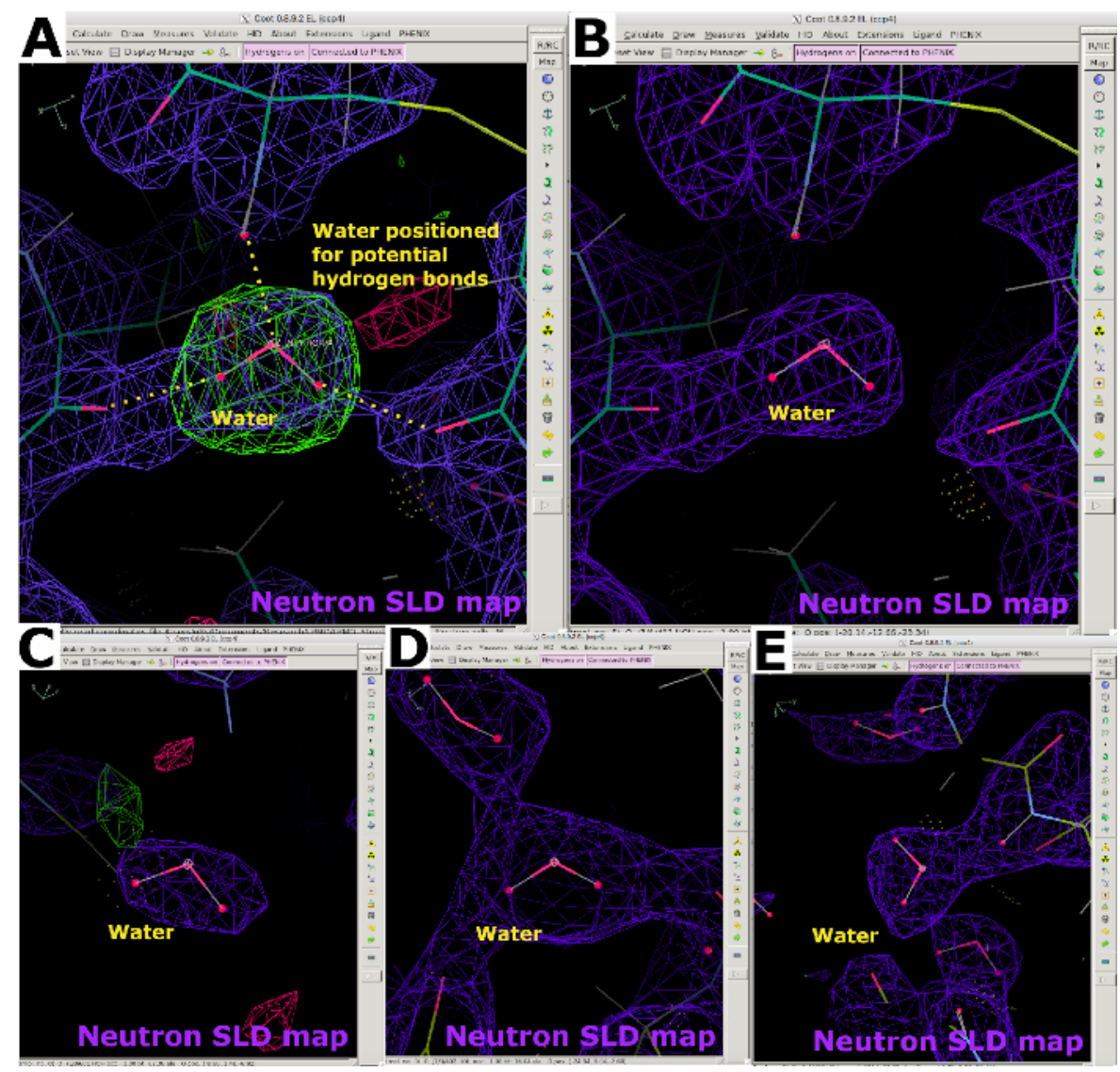

Figure 11: Appearance of water molecules in neutron SLD maps. (A) Water molecules are positioned according to FOFC neutron SLD maps (green) and potential hydrogen bonds. The 2FO-FC neutron SLD map is displayed in purple. (B) Correctly positioned water molecule. (C-E) The various shapes of neutron SLD maps for water molecules depending on Bfactors and hydrogen bond interactions. Please click here to view a larger version of this figure. 

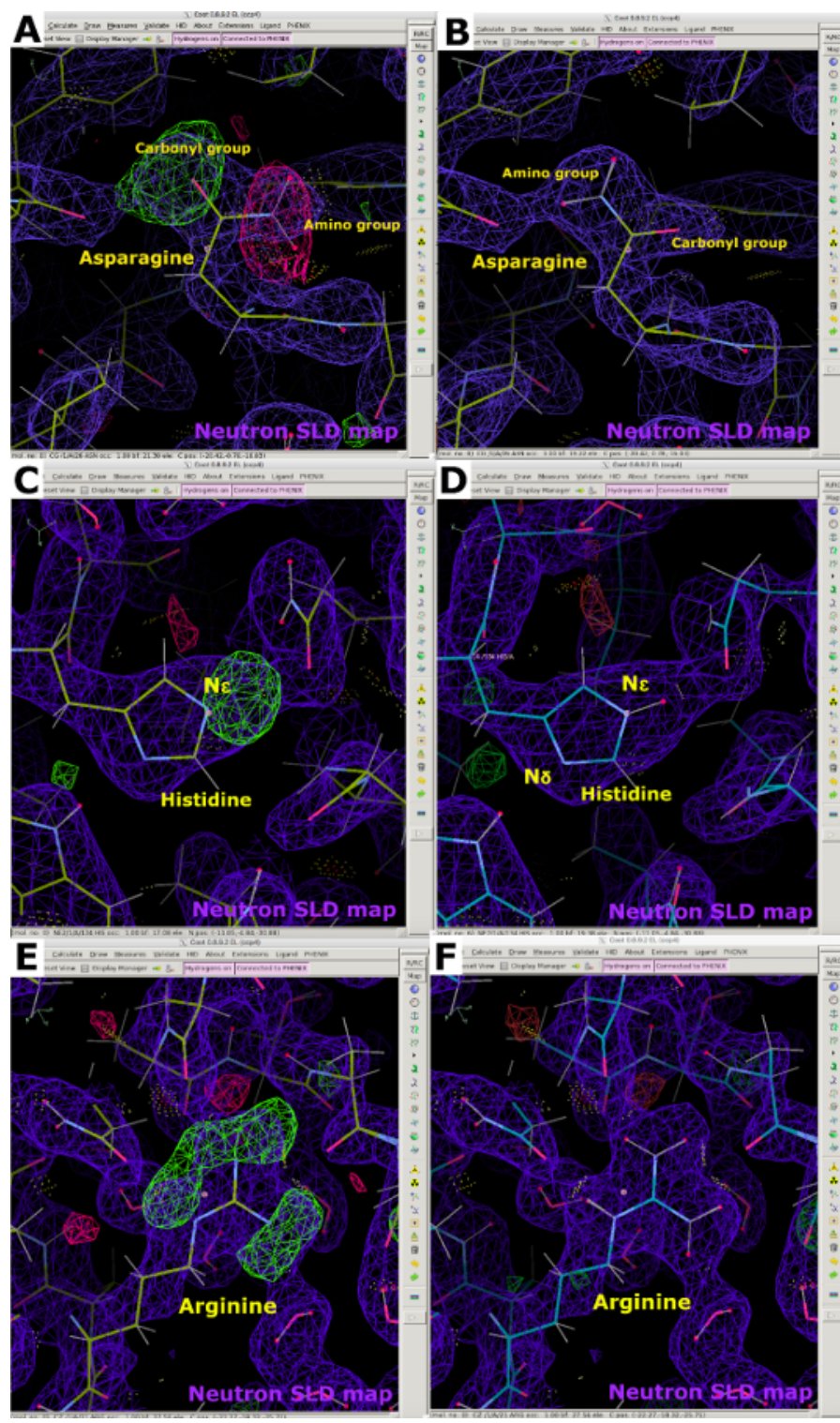

Figure 12: Information about amino acid orientation and protonation provided by neutron SLD maps. (A) The neutron SLD FO-FC map peaks (green) indicate incorrect orientation of an asparagine residue. The 2FO-FC neutron SLD map is displayed in purple and 2FO-FC electron density map is displayed in blue. (B) 2Fo-FC neutron SLD map (purple) of the correct asparagine orientation. (C) The neutron SLD FO-FC map peak (green) indicates single protonation of the histidine at $\mathrm{N}_{\varepsilon}$. (D) 2FO-FC neutron SLD map (purple) of histidine $\mathrm{N}_{\varepsilon}$-protonation. (E) Neutron SLD FO-FC omit map peaks (green) confirm the positive charge of arginine. (F) 2FO-FC neutron SLD map (purple) of positively charged arginine. Please click here to view a larger version of this figure. 

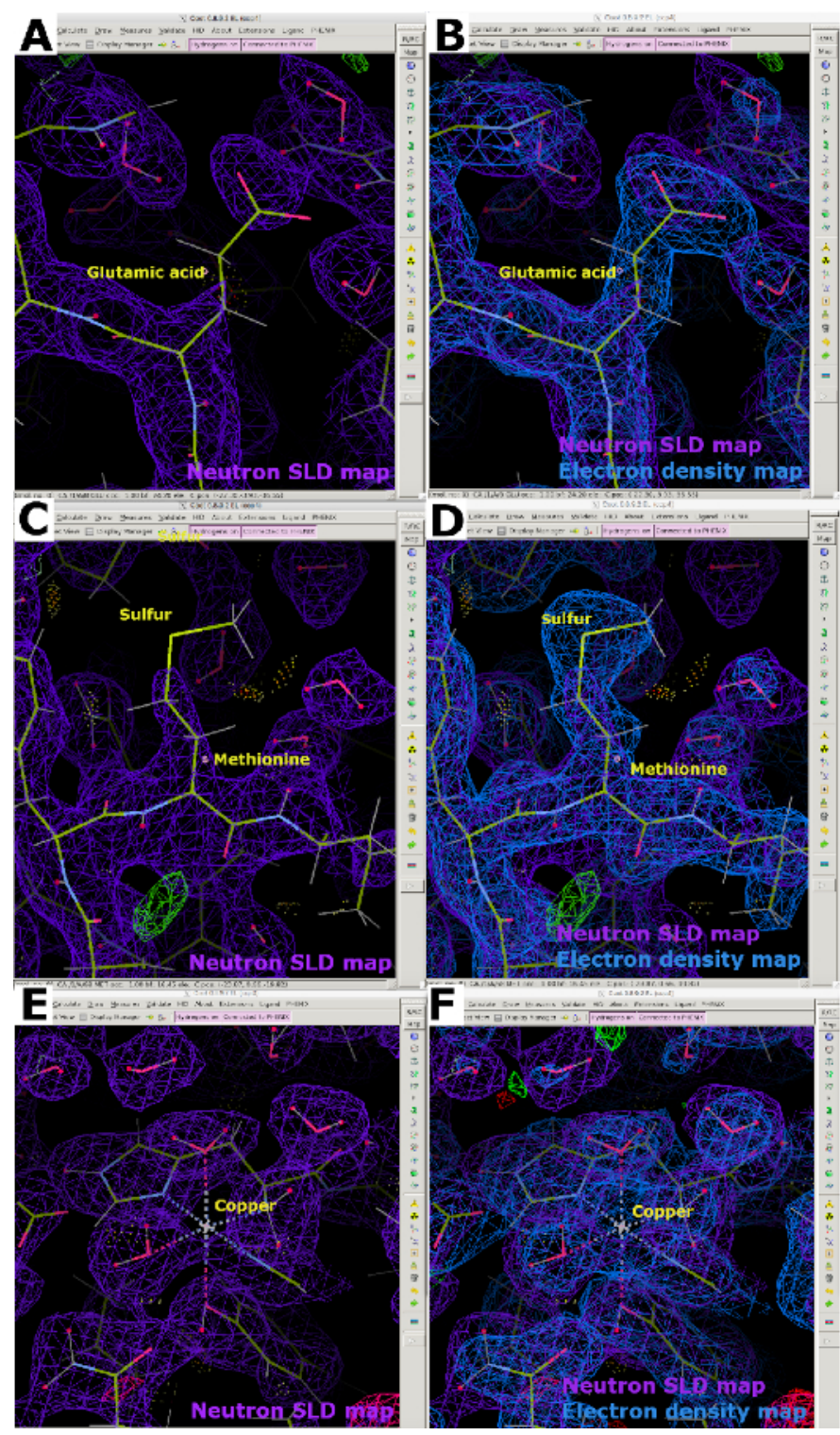

Figure 13: Discontinuous neutron SLD maps. (A) 2FO-FC neutron SLD map (purple) of a hydrogenated, vapor H/D exchanged protein. Glutamic acid displays neutron SLD map cancellation due to the negative scattering length of nonexchangeable $\mathrm{H}$ atoms. (B) An overlayed $2 \mathrm{Fo}-\mathrm{FC}_{\mathrm{C}}$ electron density map (blue) clearly displays the density of the glutamic acid. (C) The sulfur atom in methionine is poorly visible in 2FO-FC neutron SLD maps (purple). (D) An overlayed electron density map clearly displays the density of the methionine. (E) Metal atoms, here copper, are poorly visible in neutron 2FOFC SLD maps (purple). (F) An overlayed 2FO-FC electron density map (blue) clearly displays the density of the coordinated copper atom. Please click here to view a larger version of this figure. 


\begin{tabular}{|c|c|c|}
\hline Isotope & Coherent scattering length (fm) & Incoherent scattering length (fm) \\
\hline $1 \mathrm{H}$ & -3.741 & 25.274 \\
\hline $2 \mathrm{H}$ & 6.671 & 4.04 \\
\hline $12 \mathrm{C}$ & 6.6511 & 0 \\
\hline $14 \mathrm{~N}$ & 9.37 & 2 \\
\hline 160 & 5.803 & 0 \\
\hline $23 \mathrm{Na}$ & 3.63 & 3.59 \\
\hline $24 \mathrm{Mg}$ & 5.66 & 0 \\
\hline $31 P$ & 5.13 & 0.2 \\
\hline $32 S$ & 2.804 & 0 \\
\hline $35 \mathrm{Cl}$ & 11.65 & 6.1 \\
\hline $39 K$ & 3.74 & 1.4 \\
\hline $40 \mathrm{Ca}$ & 4.8 & 0 \\
\hline $55 \mathrm{Mn}$ & -3.73 & 1.79 \\
\hline $56 \mathrm{Fe}$ & 9.94 & 0 \\
\hline $63 \mathrm{Cu}$ & 6.43 & 0.22 \\
\hline $64 \mathrm{Zn}$ & 5.22 & 0 \\
\hline
\end{tabular}

Table 1: Neutron scattering lengths and incoherent scattering values. Adapted from Sears, $1992^{16}$.

Supplementary Figure 1: The IMAGINE Instrument at the

High Flux Isotope Reactor. (A) The IMAGINE instrument in the cold neutron guide hall. (B) Sample in mounted in a quartz capillary attached with putty to the goniometer. The sample and detector table closes to position the crystal and the cylindrical image plate in the neutron beam. Modified with permission of the International Union of Crystallography ${ }^{53}$. Images provided with permission of Genevieve Martin, Oak
Ridge National Laboratory. Please click here to download this figure.

Supplementary Figure 2: The MaNDi Instrument at the Spallation Neutron Source. (A) The MaNDi Anger camera detector array. Reproduced with the permission of International Union of Crystallography ${ }^{11}$. (B) MaNDi moveable sample stage. (C) Sample mounted in quartz capillary mounted on the goniometer at MaNDi for room temperature data collection. Images provided with permission 
of Genevieve Martin, Oak Ridge National Laboratory. Please click here to download this figure.

\section{Supplementary Figure 3: Structure of the lytic} polysaccharide monooxygenase NcLPMO9D. The NcLPMO9D copper-active site is located on a flat polysaccharide binding surface. The copper is coordinated by two histidine residues in a classical "histidine brace" as well as an axial tyrosine residue. Please click here to download this figure.

Supplementary Figure 4: Crystal with sufficient volume in sitting drop crystallization tray. (A) Large crystals are grown in sitting drops set up in 9-well siliconized glass plates. (B and C) Crystals are measured to identify those with volume $>0.1 \mathrm{~mm}^{3}$. Please click here to download this figure.

Supplementary Figure 5: pH meter set up for deuterated buffer readings. The $\mathrm{pH}$ electrode is soaked in $\mathrm{D}_{2} \mathrm{O}$ prior to use. $\mathrm{NaOD}$ and $\mathrm{DCl}$ are used to adjust the $\mathrm{pH}$ of deuterated buffers. Please click here to download this figure.

\section{Supplementary Figure 6: MaNDi sample mounting} guidelines. Maximum dimensions of the quartz capillary and sample position for room temperature data collection.

Reproduced from: https://neutrons.ornl.gov/mandi/sampleenvironment Please click here to download this figure.

\section{Supplementary Figure 7: Removal of excess buffer. (A)} Excess buffer is aspirated from the quartz capillary with microcapillary tips. (B) The remaining buffer is removed with a thin paper wick to completely dry the capillary. Please click here to download this figure.
Supplementary Figure 8: The data acquisition GUI. Input window of the "Experiment Parameters" for data collection. Please click here to download this figure.

Supplementary Figure 9: The Optics GUI. Selection of the quasi-Laue range for data collection and monitoring of the neutron count rate. Please click here to download this figure.

Supplementary Figure 10: Data collection in the data acquisition GUI. The exposure time, number of frames and angles for data collection are specified in the "Collect" tab. Data collection is then initiated using "Start Scan". Please click here to download this figure.

Supplementary Figure 11: Diffracted neutrons detected and displayed. At the end of the exposure time, the neutron sensitive image plate detector is read out and the diffraction pattern is displayed in the data acquisition GUI. Please click here to download this figure.

Supplementary Figure 12: Data processing following neutron diffraction. Frames are indexed, integrated, wavelength normalized and scaled using Lauegen, Lscale and Scala to generate a merged reflection file following data collection. Please click here to download this figure.

Supplementary Figure 13: X-ray data collection. Home source X-ray generator set up with quartz capillary mounted crystal for room temperature data collection. Please click here to download this figure.

\section{Supplementary Figure 14: Mounting guidelines for MaNDi} cryo-data collection. Dimensions of CrystalCaps and pin height for cryo-data collection at MaNDi. 
Reproduced from: https://neutrons.ornl.gov/mandi/sampleenvironment Please click here to download this figure.

\section{Supplementary Figure 15: Flash freezing for cryo neutron} diffraction data collection. (A) Setup for crystal soaking, harvesting with a microloop and freezing in liquid nitrogen using a cryo compatible container such as a foam Dewar. The mounted crystal is transferred directly onto the beamline cryo goniometer using precooled cryo pin tongs. (B) The wax seal is melted for crystal removal. (C) The crystal is flushed to the end of the quartz capillary for harvesting. (D) The crystal is sequentially soaked in ascorbate soak buffer and then cryoprotectant followed by flash freezing in liquid nitrogen. Please click here to download this figure.

\section{Supplementary Figure 16: Sample alignment interface.} Crystal alignment in the neutron beam, represented by the blue cross, is done by point and click centering. Please click here to download this figure.

\section{Supplementary Figure 17: The CSS GUI for data} collection. The data collection strategy, including exposure doses and angles, are uploaded in the CSS GUI. As data collection proceeds the diffracted neutrons detected on the real-time detector will be displayed in the upper panel. Please click here to download this figure.

\section{Supplementary Figure 18: Matching R-free flags in CCP4.} The R-free flags of the neutron data are matched with the Rfree flags of X-ray data collected on the same or an identical crystal for joint refinement. Please click here to download this figure.

\section{Supplementary Figure 19: Structure preparation and} refinement. (A) The Phenix ReadySet tool is used to add dual $H / D$ occupancy at exchangeable sites. (B) Both the neutron data and X-ray data are used for a joint refinement, while the initial input model was refined against the X-ray dataset collected on the same crystal or an identical crystal. Please click here to download this figure.

Supplementary Figure 20: Configuration of refinement settings. The refinement model as well as the nuclear distances are configured for joint X-ray/neutron data refinement. Please click here to download this figure.

\section{Supplementary Figure 21: Data selection for Coot model} building. The phenix MTZ file output containing X-ray and unfilled neutron data is opened in Coot to generate electron and neutron SLD maps for interactive model building. Please click here to download this figure.

Supplementary Figure 22: Interactive model building in Coot during a joint refinement. (A) A positive and negative FO-FC neutron SLD density peak (green and red, respectively) indicating that the water must be reoriented by rotation/translation. The $2 \mathrm{FO}_{\mathrm{O}} \mathrm{FC}_{\mathrm{C}}$ neutron SLD map is displayed in purple and $2 \mathrm{FO}_{\mathrm{O}}-\mathrm{FC}_{\mathrm{C}}$ electron density map is displayed in blue. (B) Correctly positioned water. (C) A positive FO-FC neutron SLD map peak (green) indicate that threonine must be rotated to match difference density peak by editing chi angles. (D) Correctly oriented threonine. Please click here to download this figure.

\section{Supplementary Figure 23: Structure preparation for} neutron-only data refinement. The starting coordinate file is prepared for refinement by water atom removal in PDBTools 
and by addition of dual H/D occupancy at exchangeable sites. Please click here to download this figure.

Supplementary Figure 24: Neutron data-only refinement. (A) Neutron data is uploaded as well as the prepared starting model. (B) The settings for neutron data refinement use the neutron scattering table. Please click here to download this figure.

Supplementary Figure 25: Data selection for Coot model building. The unfilled neutron data are opened in Coot for interactive model building. Please click here to download this figure.

Supplementary Figure 26: Real space refinement in Coot for deuterated residues. (A) Positive and negative $\mathrm{FO}_{\mathrm{O}} \mathrm{FC}_{\mathrm{C}}$ neutron SLD density peaks (green and red, respectively) indicating that an arginine residue must be moved to fit the FO-FC density peak. The 2Fo-FC neutron SLD map is displayed in purple and $2 \mathrm{FO}_{\mathrm{O}} \mathrm{FC}$ electron density map is displayed in blue. (B) Utilizing Real Space Refine results in "exploding" D atoms due to missing Coot geometry restraint libraries. (C) The $D$ atoms do not move with the rest of the residue atoms. (D) The $D$ atom positions can be manually fixed using a text editor. Please click here to download this figure.

\section{Supplementary Figure 27: Addition of water molecules.}

(A) Water molecules can be manually added to the positive FO-FC neutron SLD map density peaks (green). The inserted water molecules will initially be represented by an $\mathrm{O}$ atom in Coot. (B) Phenix ReadySet is used to add D atoms to the $\mathrm{O}$ atoms for water molecules. (C) The deuterated water molecule is successfully added. Please click here to download this figure.

Supplementary Figure 28: Refinement statistics. Final data refinement statistics following joint X-ray/neutron refinement. Please click here to download this figure.

Supplementary Figure 29: Refinement statistics. Final data refinement statistics following neutron data-only refinement. Please click here to download this figure.

\section{Discussion}

Neutron protein crystallography is a highly sensitive technique to probe protonation states and water molecule orientation in proteins. This information sheds light on protein catalytic mechanisms since changes in protonation and hydrogen bonding interactions are often central to enzyme chemistry ${ }^{10}$. Neutron protein crystallography, albeit an informative technique, has a number of factors that should be taken into consideration before planning to conduct a neutron diffraction experiment, namely:

1. The requirement for large protein crystals for data collection.

2. The scattering properties of hydrogen and other elements, such as metal ions.

3. Limitations in the structure refinement and model building software when working with deuterated samples.

Neutron protein crystallography is a flux limited technique. In contrast to X-ray diffraction datasets, higher R-factors and lower completeness, redundancy and signal-to-noise ratios are expected for neutron datasets due to the technique inherent limitations (flux limited, quasi-Laue, longer wavelengths). Data collection of a single frame is typically 12 
- 18 hours. Success of an experiment is highly dependent on sample size and quality with crystals of $0.1 \mathrm{~mm}^{3}$ often being the minimum requirement ${ }^{3}$. Neutron diffraction requires production of large amounts of protein to set up crystallization drops ranging from 10 to $800 \mu \mathrm{L}$. The minimum volume for growing sufficiently large crystals can be estimated using a Volume Calculator given the crystal and sample parameters (https://neutrons.ornl.gov/imagine). Growth of large crystals has most prevalently been accomplished by vapor diffusion ${ }^{3}$. Hanging drop crystallization permits growth of crystals in large drops ranging from 10-25 $\mu \mathrm{L}$, while larger drops ranging up to $\sim 50 \mu \mathrm{L}$ can be set up using commercially available sitting drop equipment ${ }^{14,54}$. Siliconized nine-well glass plates can be used to set up very large drops, with volumes up to $800 \mu \mathrm{L}$. These glass plates are placed in "sandwich boxes" commercially available from Hampton Research. Further crystallization techniques include batch crystallization, in which the limit of the drop size is dictated by the vessel. Batch crystallization experiment set up can range from microliters to milliliters ${ }^{55}$. Crystallization can also be performed using the dialysis technique in which the protein is equilibrated with the precipitant via a dialysis membrane or by counterdiffusion along a precipitant concentration gradient or through a porous plug such as agarose ${ }^{56,57}$. Seeding offers another alternative to obtain crystals of the desired volume. Microand macroseeding have been successfully employed for large crystal growth, including large crystal of NCLPMO9D ${ }^{45}$. Some knowledge of the protein phase diagram, including the influence of temperature on solubility, aid in large crystal growth.

When planning a neutron diffraction experiment, optimization of the protein preparation to maximize signal-to-noise ratio during diffraction data collection is essential ${ }^{7}$. To circumvent density cancellation and high incoherent scattering caused by $\mathrm{H}$ atoms, neutron SLD maps can be improved by exchanging $\mathrm{H}$ atoms for its isotope $\mathrm{D}$, which possesses a positive coherent scattering length and low incoherent scattering length. To accomplish this, vapor exchange of the hydrogenated protein crystal against deuterated crystallization buffer is performed. This ensures $H / D$ exchange of solvent molecules and the labile, titratable protein $\mathrm{H}$ atoms ${ }^{23}$. Vapor exchange is performed by mounting the hydrogenated crystal in a quartz capillary with $\mathrm{D}_{2} \mathrm{O}$-based, deuterated crystallization buffer "plugs" and it represents an effective, gentle technique that is most often applied ${ }^{14,23,35}$. The exchange can take several weeks and preferably requires the deuterated buffer to be frequently changed to ensure maximum $H / D$ exchange. $H /$ D exchange can also be performed by directly soaking the crystal in deuterated buffer. To avoid placing the crystal under stress due to $\mathrm{D}_{2} \mathrm{O}$ exposure, the soaking process should be performed gradually by incrementally increasing the $\mathrm{D}_{2} \mathrm{O}: \mathrm{H}_{2} \mathrm{O}$ ratio ${ }^{58}$. In addition to this, crystallization of hydrogenated protein can also be performed in deuterated buffer for $H / D$ exchange at labile $H$ sites $^{22,59}$. It should be noted, however, that $\mathrm{D}_{2} \mathrm{O}$-based buffer has an effect on protein solubility requiring further adjustment of the known $\mathrm{H}_{2} \mathrm{O}$-based conditions ${ }^{3,59} \cdot \mathrm{D}_{2} \mathrm{O}$-based buffers have also been observed to lead to smaller crystals in some cases ${ }^{59}$. Full exchange of titratable and carbon-bound $\mathrm{H}$ atoms to $\mathrm{D}$ can be achieved by expressing proteins in deuterated media to generate a perdeuterated sample ${ }^{20}$. The resulting neutron SLD maps of the perdeuterated sample will be significantly improved, no longer displaying the density cancellation of the hydrogenated sample counterpart. This is beneficial when characterizing $H / D$ bound at non-exchangeable sites in a protein or cofactor. However, expression of perdeuterated protein is both high in cost and low in yield ${ }^{60}$. The Oak Ridge National Laboratory (ORNL) Center for Structural 
Molecular Biology (CSMB) offers a deuteration facility for users seeking to generate a perdeuterated sample (https:// www.ornl.gov/facility/csmb). Perdeuterated expression is typically performed in a bioreactor on the $1 \mathrm{~L}$ scale yielding $\sim 50 \mathrm{mg}$ of purified protein ${ }^{61}$.

Following the collection of neutron diffraction data, refinement and interactive model building is performed. Refinement can be run using multiple software suites including phenix.refine, nCNS or SHELXL $28,31,32,33$. The Phenix suite is the most commonly utilized software for refinement of neutron diffraction data in conjunction with Coot which is used to manually build the model from the neutron SLD maps ${ }^{34}$. Although both Phenix and Coot allow for the processing of neutron diffraction data, they may lack certain features necessary to process the idiosyncrasies associated with neutron data and deuterated samples. For example, Coot does not contain geometry optimization for deuterated residues, which can lead to complications during model building since the "Real Space Refine" feature results in "exploding" residues (Supplementary Figure 26) ${ }^{62}$. This can be resolved by generating restraint files for all the deuterated residues. However, this is an intensive process and such libraries are not currently publicly available. When performing refinements in Phenix, exchangeable $H / D$ sites will initially be set to 0.50 occupancy for $H$ and $D$. As refinements are performed, the occupancy of $H$ and $D$ will be refined according to the neutron SLD maps. During interactive model building, difference density $\mathrm{F}_{\mathrm{O}}-\mathrm{F}_{\mathrm{C}}$ maps are very informative in assessing H/D occupancies. Maps can be used to determine which sites possess high D occupancy, which is particularly informative at the active site where protonation states are catalytically relevant ${ }^{63}$. Ambiguous situations do arise, however, when the H:D occupancy is close to 0.70:0.30 which results in complete signal cancellation in neutron SLD maps $^{64}$. It should also be taken into account that quasi-Laue neutron data sets often have completeness around $80 \%$, which is lower than the routinely observed $\geq 98 \%$ for $X$ ray diffraction data. When refining neutron diffraction data in Phenix, the missing observed amplitudes $\left(F_{0}\right)$ are therefore calculated from the model to complete the reflection list, thus introducing model bias. To account for this potential bias "no_fill" maps should be examined during interactive model building as opposed to "filled" maps.

Users can choose to perform a joint X-ray/neutron data refinement of their structure, or a neutron-data only refinement. Visualizing neutron SLD maps, particularly at lower resolution, may initially be disconcerting especially for a hydrogenated protein in which $\mathrm{H}$ is still present at nonexchangeable sites despite H/D vapor exchange. This results in neutron density map cancellations, giving the impression of discontinuous maps ${ }^{65,66}$. Collecting a corresponding X-ray dataset advantageously complements these cancellations in a joint refinement (Figure 13A and Figure 13B). A jointrefinement strategy typically involves refining the protein backbone coordinates against the $\mathrm{X}$-ray data, while the neutron diffraction data is used to refine the position and occupancy of the $H / D$ atoms at exchangeable $\operatorname{sites}^{28}$. Since introduction of joint H/D occupancy at exchangeable sites increases the number of parameters being refined, a joint refinement with $\mathrm{X}$-ray data also increases the data-toparameter ratio. A joint refinement requires a corresponding X-ray dataset to be collected at the same temperature on the same crystal or a crystal grown under the same conditions. For neutron diffraction data collected at room temperature (300K), the corresponding X-ray dataset should be collected at room temperature using a low-dose data collection strategy to limit radiation damage. Perdeuterated samples, in contrast, provide improved and continuous neutron SLD maps since 
they do not possess the same magnitude of $H / D$ signal cancellation. However, the neutron scattering length of certain elements including metals and sulfur make them poorly visible in neutron SLD maps, even if the protein has been perdeuterated (Figure $13 \mathrm{C}-\mathbf{F})^{18}$. If a metal needs to be characterized, it is best to utilize X-ray diffraction in a joint refinement or apply spectroscopic techniques to complement diffraction experiments. Neutron-only data refinements are often performed when the neutron dataset has high resolution or if a perdeuterated protein was used. In addition, neutrononly data refinement is particularly useful if a protein highly sensitive to radiation damage is being studied, since an X-ray derived structure may possess radiation-induced artefacts. If a neutron-data-only refinement is to be performed, it must be ascertained whether the corresponding neutron dataset has sufficient completeness and resolution.

ORNL offers two facilities for collection of neutron diffraction data: the IMAGINE beamline at the HFIR as well as the MaNDi beamline at the $\mathrm{SNS}^{36,67}$. While both instruments provide effective means for collecting a neutron diffraction dataset employing similar principles, each instrument has unique specifications that should be taken into account when applying for beam time. IMAGINE collects quasi-Laue data and is optimized for room temperature data collection on crystals with unit cells up to $\sim 100 \AA$. MaNDi can be used for the collection of room temperature and cryo-temperature data employing TOF-Laue collection on crystals with unit cells up to $\sim 300 \AA$. Prior to collecting a complete dataset, a test is performed on the crystal to evaluate the quality of the obtained diffraction pattern in which the crystal is exposed to the neutron beam for a single frame. If the crystal is of sufficient quality, a full neutron diffraction dataset will be collected, indexed, integrated, scaled and merged in a process analogous to X-ray data processing. IMAGINE makes use of Lauegen and Lscale and MaNDi utilizes the Mantid package and employs three-dimensional profile fitting $48,50,51,68,69,70$. Scientists who become users at either of these facilities will be provided with a dataset in MTZ or HKL format for further analysis.

Neutron diffraction is a non-destructive, highly sensitive technique for probing the protonation state and hydrogen bond interactions of biological macromolecules. It is particularly useful for photo-sensitive proteins and metalloproteins. Several considerations regarding the technique as well as the processing of the data must be taken into account before conducting an experiment, however the outcome yields results which may give valuable insight into the catalytic mechanism of the protein of interest. Neutron protein crystallography complements computational, structural, biochemical and spectroscopic studies, making it a valuable tool in the biologist's toolbox of techniques used to characterize biological macromolecules.

\section{Disclosures}

The authors have nothing to disclose.

\section{Acknowledgments}

Protein expression, purification and crystallization experiments were conducted at the Center for Structural Molecular Biology (CSMB), a U.S. Department of Energy Biological and Environmental Research User Facility at Oak Ridge National Laboratory. Neutron diffraction data was collected at BL-11B MaNDi at the Spallation Neutron Source (SNS) at ORNL which is sponsored by the Scientific User Facilities Division, Office of Basic Energy Sciences, U.S. Department of Energy. The authors thank Brendan Sullivan for assistance with data reduction. X-ray diffraction data was collected at the Molecular Education, Technology, and 
Research Innovation Center (METRIC) facilities at North Carolina State University, which is supported by the State of North Carolina. GCS acknowledges support in part from the National Research Foundation (NRF), South Africa and the Graduate Opportunities (GO!) program at ORNL. FM acknowledges support from USDA NIFA Hatch 211001.

\section{References}

1. Neumann, P., Tittmann, K. Marvels of enzyme catalysis at true atomic resolution: distortions, bond elongations, hidden flips, protonation states and atom identities. Current Opinion in Structural Biology. 29, 122-133 (2014).

2. Pynn, R. Neutron Scattering-A Non-destructive Microscope for Seeing Inside Matter. Neutron Applications in Earth, Energy and Environmental Sciences. 15-36 (2009).

3. O'Dell, W.B., Bodenheimer, A.M., Meilleur, F. Neutron protein crystallography: A complementary tool for locating hydrogens in proteins. Archives of Biochemistry and Biophysics. 602, 48-60 (2016).

4. Niimura, N., Podjarny, A. Neutron Protein Crystallography: Hydrogen, Protons, and Hydration in Bio-macromolecules. Oxford University Press. Oxford, UK. (2011).

5. Blakeley, M.P.P. Neutron macromolecular crystallography. Crystallography Reviews. 15 (3), 157-218 (2009).

6. Blakeley, M.P., Cianci, M., Helliwell, J.R., Rizkallah, P.J. Synchrotron and neutron techniques in biological crystallography. Chemical Society Reviews. 33 (8), 548-557 (2004).
7. Ashkar, R. et al. Neutron scattering in the biological sciences: progress and prospects. Acta Crystallographica Section D: Structural Biology. 74 (12) (2018).

8. Teixeira, S.C.M. et al. New sources and instrumentation for neutrons in biology. Chemical Physics. 345 (2-3), 133-151 (2008).

9. Furrer, A., Mesot, J., Strässle, T. Neutron Scattering in Condensed Matter Physics. World Scientific Publishing Company. Singapore (2009).

10. Meilleur, F., Coates, L., Cuneo, M.J., Kovalevsky, A., Myles, D.A.A. The neutron macromolecular crystallography instruments at Oak Ridge national laboratory: Advances, challenges, and opportunities. Crystals. 8 (10), 1-10 (2018).

11. Coates, L. et al. The Macromolecular Neutron Diffractometer MaNDi at the Spallation Neutron Source. Journal of Applied Crystallography. 48, 1302-1306 (2015).

12. Coates, L., Stoica, A.D., Hoffmann, C., Richards, J., Cooper, R. The macromolecular neutron diffractometer (MaNDi) at the Spallation Neutron Source, Oak Ridge: enhanced optics design, high-resolution neutron detectors and simulated diffraction. Journal of Applied Crystallography. 43 (3), 570-577 (2010).

13. Koetzle, T.F., Piccoli, P.M.B., Schultz, A.J. Single-crystal neutron diffraction studies of hydrogen-bonded systems: Two recent examples from IPNS. Nuclear Instruments and Methods in Physics Research, Section A: Accelerators, Spectrometers, Detectors and Associated Equipment. 600 (1), 260-262 (2009). 
14. Ng, J.D. et al. Large-volume protein crystal growth for neutron macromolecular crystallography. Acta Crystallographica Section F:Structural Biology Communications. 71, 358-370 (2015).

15. Blakeley, M.P., Langan, P., Niimura, N., Podjarny, A. Neutron crystallography: opportunities, challenges, and limitations. Current Opinion in Structural Biology. 18 (5), 593-600 (2008).

16. Sears, V.F. Neutron scattering lengths and cross sections. Neutron News. 3 (3), 26-37 (1992).

17. Weik, M., Patzelt, H., Zaccai, G., Oesterhelt, D. Localization of glycolipids in membranes by in vivo labeling and neutron diffraction. Molecular Cell. 1 (3), 411-419 (1998).

18. Helliwell, J.R. Fundamentals of neutron crystallography in structural biology. Methods in Enzymology. 634 Elsevier Inc. (2020).

19. Niimura, N., Bau, R. Neutron protein crystallography: Beyond the folding structure of biological macromolecules. Acta Crystallographica Section A: Foundations of Crystallography. 64 (1), 12-22 (2008).

20. Hazemann, I. et al. High-resolution neutron protein crystallography with radically small crystal volumes: Application of perdeuteration to human aldose reductase. Acta Crystallographica Section D: Biological Crystallography. 61 (10), 1413-1417 (2005).

21. Niimura, N., Chatake, T., Ostermann, A., Kurihara, K., Tanaka, I. High resolution neutron protein crystallography. Hydrogen and hydration in proteins. Zeitschrift für Kristallographie - Crystalline Materials. 218 (2) (2003).
22. Meilleur, F., Contzen, J., Myles, D.A.A., Jung, C. Structural stability and dynamics of hydrogenated and perdeuterated cytochrome P450cam (CYP101). Biochemistry. 43 (27), 8744-8753 (2004).

23. Bennett, B.C., Gardberg, A.S., Blair, M.D., Dealwis, C.G. On the determinants of amide backbone exchange in proteins: A neutron crystallographic comparative study. Acta Crystallographica Section D: Biological Crystallography. 64 (7), 764-783 (2008).

24. Meilleur, F., Kovalevsky, A., Myles, D.A.A. IMAGINE: The neutron protein crystallography beamline at the high flux isotope reactor. Methods in Enzymology. 634, Elsevier Inc. (2020).

25. Wang, X.P. et al. A suite-level review of the neutron single-crystal diffraction instruments at Oak Ridge National Laboratory. Review of Scientific Instruments. 89 (9), 092802 (2018).

26. Wlodawer, A., Hendrickson, W.A. A procedure for joint refinement of macromolecular structures with X-ray and neutron diffraction data from single crystals. Acta Crystallographica Section A. 38 (2), 239-247 (1982).

27. Halle, B. Biomolecular cryocrystallography: Structural changes during flash-cooling. Proceedings of the National Academy of Sciences of the United States of America. 101 (14), 4793-4798 (2004).

28. Adams, P.D., Mustyakimov, M., Afonine, P. V., Langan, P. Generalized X-ray and neutron crystallographic analysis: More accurate and complete structures for biological macromolecules. Acta Crystallographica Section D: Biological Crystallography. 65 (6), 567-573 (2009). 
29. Berman, H.M. et al. The Protein Data Bank. Acta Crystallographica Section D Biological Crystallography. 58 (6), 899-907 (2002).

30. Liebschner, D., Afonine, P. V., Moriarty, N.W., Langan, P., Adams, P.D. Evaluation of models determined by neutron diffraction and proposed improvements to their validation and deposition. Acta Crystallographica Section D: Structural Biology. 74, 800-813 (2018).

31. Afonine, P. V., Mustyakimov, M., Grosse-Kunstleve, R.W., Moriarty, N.W., Langan, P., Adams, P.D. Joint X-ray and neutron refinement with phenix.refine. Acta Crystallographica Section D: Biological Crystallography. 66 (11), 1153-1163 (2010).

32. Brünger, A.T. et al. Crystallography \& NMR system: A new software suite for macromolecular structure determination. Acta Crystallographica Section D: Biological Crystallography. 54 (5), 905-921 (1998).

33. Gruene, T., Hahn, H.W., Luebben, A. V., Meilleur, F., Sheldrick, G.M. Refinement of macromolecular structures against neutron data with SHELXL2013. Journal of Applied Crystallography. 47 (1), 462-466 (2014).

34. Emsley, P., Lohkamp, B., Scott, W.G., Cowtan, K. Features and development of Coot. Acta Crystallographica Section D Biological Crystallography. 66 (4), 486-501 (2010).

35. Lakey, J.H. Neutrons for biologists: A beginner's guide, or why you should consider using neutrons. Journal of the Royal Society Interface. 6 (SUPPL. 5) (2009).

36. Schröder, G.C., O’Dell, W.B., Myles, D.A.A., Kovalevsky, A., Meilleur, F. IMAGINE: Neutrons reveal enzyme chemistry. Acta Crystallographica Section D: Structural Biology. 74, 778-786 (2018).

37. Halsted, T.P. et al. Catalytically important damage-free structures of a copper nitrite reductase obtained by femtosecond X-ray laser and room-temperature neutron crystallography. IUCrJ. 6, 761-772 (2019).

38. Meier, K.K. et al. Oxygen Activation by Cu LPMOs in Recalcitrant Carbohydrate Polysaccharide Conversion to Monomer Sugars. Chemical Reviews. 118 (5), 2593-2635 (2018).

39. Beeson, W.T., Vu, V. V., Span, E.A., Phillips, C.M., Marletta, M.A. Cellulose Degradation by Polysaccharide Monooxygenases. Annual Review of Biochemistry. 84 (1), 923-946 (2015)

40. Walton, P.H., Davies, G.J. On the catalytic mechanisms of lytic polysaccharide monooxygenases. Current Opinion in Chemical Biology. 31, 195-207 (2016).

41. Bertini, L. et al. Catalytic Mechanism of Fungal Lytic Polysaccharide Monooxygenases Investigated by FirstPrinciples Calculations. Inorganic Chemistry. 57 (1), 86-97 (2018).

42. Hedegård, E.D., Ryde, U. Molecular mechanism of lytic polysaccharide monooxygenases. Chemical Science. 9 (15), 3866-3880 (2018).

43. Hangasky, J.A., Detomasi, T.C., Marletta, M.A. Glycosidic Bond Hydroxylation by Polysaccharide Monooxygenases. Trends in Chemistry. 1 (2), 198-209 (2019).

44. Bacik, J.P. et al. Neutron and Atomic Resolution X-ray Structures of a Lytic Polysaccharide Monooxygenase Reveal Copper-Mediated Dioxygen Binding and 
Evidence for N-Terminal Deprotonation. Biochemistry. 56 (20), 2529-2532 (2017).

45. O'Dell, W.B., Agarwal, P.K., Meilleur, F. Oxygen Activation at the Active Site of a Fungal Lytic Polysaccharide Monooxygenase. Angewandte Chemie International Edition. 56 (3), 767-770 (2017).

46. O'Dell, W.B., Swartz, P.D., Weiss, K.L., Meilleur, F. Crystallization of a fungal lytic polysaccharide monooxygenase expressed from glycoengineered Pichia pastoris for X-ray and neutron diffraction. Acta Crystallographica Section:F Structural Biology Communications. 73 (2), 70-78 (2017).

47. Meilleur, F. et al. The IMAGINE instrument: First neutron protein structure and new capabilities for neutron macromolecular crystallography. Acta Crystallographica Section D: Biological Crystallography. 69 (10), 2157-2160 (2013).

48. Helliwell, J.R. et al. The recording and analysis of synchrotron X-radiation Laue diffraction photographs. Journal of Applied Crystallography. 22 (5), 483-497 (1989).

49. Nieh, Y.P. et al. Accurate and highly complete synchrotron protein crystal Laue diffraction data using the ESRF CCD and the Daresbury Laue software. Journal of Synchrotron Radiation. 6 (5), 995-1006 (1999).

50. Arzt, S., Campbell, J.W., Harding, M.M., Hao, Q., Helliwell, J.R. LSCALE - The new normalization, scaling and absorption correction program in the Daresbury Laue software suite. Journal of Applied Crystallography. 32 (3), 554-562 (1999).

51. Sullivan, B. et al. Improving the accuracy and resolution of neutron crystallographic data by three-dimensional profile fitting of Bragg peaks in reciprocal space. Acta
Crystallographica Section D: Structural Biology. 74 (11), 1085-1095 (2018).

52. Sullivan, B. et al. BraggNet: integrating Bragg peaks using neural networks. Journal of Applied Crystallography. 52 (4), 854-863 (2019).

53. Schröder, G.C., O'Dell, W.B., Myles, D.A.A., Kovalevsky, A., Meilleur, F. IMAGINE: Neutrons reveal enzyme chemistry. Acta Crystallographica Section D: Structural Biology. 74 (2018).

54. Blum, M.M. et al. Preliminary time-of-flight neutron diffraction study on diisopropyl fluorophosphatase (DFPase) from Loligo vulgaris. Acta Crystallographica Section F: Structural Biology and Crystallization Communications. 63 (1), 42-45 (2007).

55. Tomanicek, S.J. et al. Neutron and X-ray crystal structures of a perdeuterated enzyme inhibitor complex reveal the catalytic proton network of the Toho-1 $\beta$ lactamase for the acylation reaction. Journal of Biological Chemistry. 288 (7), 4715-4722 (2013).

56. Metcalfe, C. et al. The tuberculosis prodrug isoniazid bound to activating peroxidases. Journal of Biological Chemistry. 283 (10), 6193-6200 (2008).

57. Hughes, R.C. et al. Inorganic pyrophosphatase crystals from Thermococcus thioreducens for $\mathrm{X}$-ray and neutron diffraction. Acta Crystallographica Section F: Structural Biology and Crystallization Communications. 68 (12), 1482-1487 (2012).

58. Bennett, B.C., Meilleur, F., Myles, D.A.A., Howell, E.E., Dealwis, C.G. Preliminary neutron diffraction studies of Escherichia coli dihydrofolate reductase bound to the anticancer drug methotrexate. Acta Crystallographica Section D: Biological Crystallography. 61 (5), 574-579 (2005). 
59. Snell, E.H. et al. Optimizing crystal volume for neutron diffraction: D-xylose isomerase. European Biophysics Journal. 35 (7), 621-632 (2006).

60. Golden, E., Attwood, P. V., Duff, A.P., Meilleur, F., Vrielink, A. Production and characterization of recombinant perdeuterated cholesterol oxidase. Analytical Biochemistry. 485, 102-108 (2015).

61. Munshi, P. et al. Rapid visualization of hydrogen positions in protein neutron crystallographic structures. Acta Crystallographica Section D: Biological Crystallography. 68 (1), 35-41 (2012).

62. Logan, D.T. Interactive model building in neutron macromolecular crystallography. Methods in Enzymology. 634. Elsevier Inc. (2020).

63. Koruza, K., Lafumat, B., Végvári, Knecht, W., Fisher, S.Z. Deuteration of human carbonic anhydrase for neutron crystallography: Cell culture media, protein thermostability, and crystallization behavior. Archives of Biochemistry and Biophysics. 645 (March), 26-33 (2018).

64. Fisher, S.J. et al. Perdeuteration: Improved visualization of solvent structure in neutron macromolecular crystallography. Acta Crystallographica Section D: Biological Crystallography. 70 (12), 3266-3272 (2014).

65. Chen, J.C. H., Hanson, B.L., Fisher, S.Z., Langan, P., Kovalevsky, a. Y. Direct observation of hydrogen atom dynamics and interactions by ultrahigh resolution neutron protein crystallography. Proceedings of the National Academy of Sciences. 109 (38), 15301-15306 (2012).

66. Cuypers, M.G. et al. Near-atomic resolution neutron crystallography on perdeuterated Pyrococcus furiosus rubredoxin: Implication of hydronium ions and protonation state equilibria in redox changes.
Angewandte Chemie - International Edition. 52 (3), 1022-1025 (2013).

67. Coates, L., Sullivan, B. The macromolecular neutron diffractometer at the spallation neutron source. Methods in Enzymology. 634, . Elsevier Inc. (2020).

68. Ren, Z., Kingman, N.G., Borgstahl, G.E.O., Getzoff, E.D., Moffat, K. Quantitative Analysis of Time-Resolved Laue Diffraction Patterns. Journal of Applied Crystallography. 29 (3), 246-260 (1996).

69. Campbell, J.W., Hao, Q., Harding, M.M., Nguti, N.D., Wilkinson, C. LAUEGEN version 6.0 and INTLDM. Journal of Applied Crystallography. 31 (3), 496-502 (1998).

70. Arnold, O. et al. Mantid - Data analysis and visualization package for neutron scattering and $\mu \mathrm{SR}$ experiments. Nuclear Instruments and Methods in Physics Research, Section A: Accelerators, Spectrometers, Detectors and Associated Equipment. 764, 156-166 (2014). 